\title{
Closing the window on single leptoquark solutions to the $B$-physics anomalies
}

\author{
A. Angelescu, ${ }^{a}$ D. Bečirević, ${ }^{b}$ D.A. Faroughy ${ }^{c}$ and O. Sumensari ${ }^{d, e}$ \\ ${ }^{a}$ Department of Physics and Astronomy, University of Nebraska-Lincoln, \\ Lincoln, NE, 68588, U.S.A. \\ ${ }^{b}$ Laboratoire de Physique Théorique (Bât. 210), \\ CNRS, Univ. Paris-Sud, Université Paris-Saclay, 91405 Orsay, France \\ ${ }^{c} J$. Stefan Institute, \\ Jamova 39, P.O. Box 3000, 1001 Ljubljana, Slovenia \\ ${ }^{d}$ Istituto Nazionale Fisica Nucleare, Sezione di Padova, \\ I-35131 Padova, Italy \\ eDipartamento di Fisica e Astronomia "G. Galilei", Università di Padova, \\ Via Francesco Marzolo 8, 35121 Padova, Italy \\ E-mail: aangelescu2@unl.edu, Damir.Becirevic@th.u-psud.fr, \\ darius.faroughy@ijs.si, olcyr.sumensari@pd.infn.it
}

Abstract: We examine various scenarios in which the Standard Model is extended by a light leptoquark state to solve for one or both $B$-physics anomalies, viz. $R_{D^{(*)}}^{\exp }>R_{D^{(*)}}^{\mathrm{SM}}$ or/and $R_{K^{(*)}}^{\exp }<R_{K^{(*)}}^{\mathrm{SM}}$. To do so we combine the constraints arising both from the lowenergy observables and from direct searches at the LHC. We find that none of the scalar leptoquarks of mass $m_{\mathrm{LQ}} \simeq 1 \mathrm{TeV}$ can alone accommodate the above mentioned anomalies. The only single leptoquark scenario which can provide a viable solution for $m_{\mathrm{LQ}} \simeq 1 \div 2 \mathrm{TeV}$ is a vector leptoquark, known as $U_{1}$, which we re-examine in its minimal form (letting only left-handed couplings to have non-zero values). We find that the limits deduced from direct searches are complementary to the low-energy physics constraints. In particular, we find a rather stable lower bound on the lepton flavor violating $b \rightarrow s \ell_{1}^{ \pm} \ell_{2}^{\mp}$ modes, such as $\mathcal{B}(B \rightarrow K \mu \tau)$. Improving the experimental upper bound on $\mathcal{B}(B \rightarrow K \mu \tau)$ by two orders of magnitude could compromise the viability of the minimal $U_{1}$ model as well.

Keywords: Beyond Standard Model, Effective Field Theories

ArXiv EPrint: 1808.08179 


\section{Contents}

1 Introduction 1

2 Effective theory description $\quad 3$

$2.1 b \rightarrow s \ell_{1}^{-} \ell_{2}^{+}$and $R_{K^{(*)}}$

$2.2 \quad b \rightarrow c \ell \bar{\nu}$ and $R_{D^{(*)}}$

3 Leptoquark models for $\boldsymbol{R}_{K^{(*)}}$ and/or $\boldsymbol{R}_{\boldsymbol{D}^{(*)}}$

$\begin{array}{lll}3.1 & \text { Scalar leptoquarks } & 7\end{array}$

$\begin{array}{lll}3.1 .1 & S_{3}=(\overline{\mathbf{3}}, \mathbf{3})_{1 / 3} & 7\end{array}$

3.1.2 $R_{2}=(\mathbf{3}, \mathbf{2})_{7 / 6} \quad 8$

3.1.3 $\widetilde{R}_{2}=(\mathbf{3}, \mathbf{2})_{1 / 6} \quad 9$

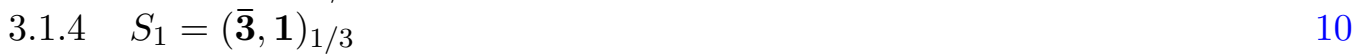

$\begin{array}{lll}3.2 & \text { Vector leptoquarks } & 10\end{array}$

$\begin{array}{lll}3.2 .1 & U_{1}=(\mathbf{3}, \mathbf{1})_{2 / 3} & 10\end{array}$

$\begin{array}{lll}3.2 .2 & U_{3}=(\mathbf{3}, \mathbf{3})_{2 / 3} & 11\end{array}$

4 High- $p_{T}$ phenomenology 12

$\begin{array}{lll}4.1 & \text { Direct limits on pair-produced LQs } & 12\end{array}$

$\begin{array}{lll}4.2 & \text { Limits from high- } p_{T} \text { tails of } p p \rightarrow \ell \bar{\ell} & 13\end{array}$

5 Which leptoquark model? $\quad 15$

6 Revisiting $U_{1}=(3,1)_{2 / 3} \quad 20$

$\begin{array}{lll}6.1 & \text { Low-energy constraints } & 20\end{array}$

$\begin{array}{ll}6.2 \text { Results and predictions } & 22\end{array}$

$\begin{array}{lll}7 & \text { Summary and conclusion } & 25\end{array}$

\section{Introduction}

Over the past several years we witnessed a growing interest in theoretical studies of the origin of lepton flavor universality violation (LFUV), motivated by a number of experimental hints in weak decays of $B$-mesons pointing towards LFUV. The first such indication was reported by BaBar in refs. [1,2] in which they measured

$$
R_{D^{(*)}}=\left.\frac{\mathcal{B}\left(B \rightarrow D^{(*)} \tau \bar{\nu}\right)}{\mathcal{B}\left(B \rightarrow D^{(*)} l \bar{\nu}\right)}\right|_{l \in\{e, \mu\}},
$$

and found an excess in $\mathcal{B}\left(B \rightarrow D^{(*)} \tau \bar{\nu}\right)$. Since that time, Belle and LHCb measured the same ratio [3-7] and observed a similar feature, namely that the measured $R_{D^{(*)}}^{\exp }$ is larger 
than $R_{D^{(*)}}^{\mathrm{SM}}$, the value predicted in the Standard Model (SM). The most recent HFLAV averages are [8]:

$$
R_{D}=0.41(5), \quad R_{D^{*}}=0.31(2),
$$

which, when combined, give $3.8 \sigma$ excess with respect to (w.r.t.) the $\mathrm{SM}$ values, $R_{D}^{\mathrm{SM}}=$ $0.300(8)$ [9-11], and $R_{D^{*}}^{\mathrm{SM}}=0.257(3)$ [12-14]. Apart from the reduction of a significant part of the systematic experimental errors, the advantage of considering the ratio of decay rates lies in the fact that the Cabibbo-Kobayashi-Maskawa (CKM) factors cancel out and in the fact that the sensitivity to hadronic uncertainties is much smaller than it is in the case with one of the branching fractions alone $\mathcal{B}\left(B \rightarrow D^{(*)} \ell \bar{\nu}\right), \ell \in\{e, \mu, \tau\}$. Even though a $5 \sigma$ significance of LFUV in the tree-level $b \rightarrow c \ell \bar{\nu}$ decay has not yet been reached, the experimentalists of $\mathrm{LHCb}$ were able to confirm the same tendency in another hadronic environment. They measured [15]

$$
R_{J / \psi}=\frac{\mathcal{B}\left(B_{c} \rightarrow J / \psi \tau \bar{\nu}\right)}{\mathcal{B}\left(B_{c} \rightarrow J / \psi \mu \bar{\nu}\right)}=0.71 \pm 0.25,
$$

which again appears to be $\approx 2 \sigma$ larger than its SM value.

Another indication of the LFUV came from the weak decays mediated by a flavor changing neutral current (FCNC), $b \rightarrow s l^{+} l^{-}$. The experimentalists of LHCb measured

$$
R_{K^{(*)}}^{\left[q_{1}^{2}, q_{2}^{2}\right]}=\frac{\mathcal{B}^{\prime}\left(B \rightarrow K^{(*)} \mu \mu\right)}{\mathcal{B}^{\prime}\left(B \rightarrow K^{(*)} e e\right)},
$$

where $\mathcal{B}^{\prime}$ stands for the partial branching fraction comprising $q^{2}=\left(p_{l^{+}}+p_{l^{-}}\right)^{2}$ between $q_{1}^{2}$ and $q_{2}^{2}$ (in units of $\mathrm{GeV}^{2}$ ). They reported $[16,17]$ :

$$
R_{K} \equiv R_{K^{+}}^{[1,6]}=0.75 \pm 0.09, \quad R_{K^{*}} \equiv R_{K^{* 0}}^{[1.1,6]}=0.71 \pm 0.10, \quad R_{K^{* 0}}^{[0.045,1.1]}=0.68 \pm 0.10
$$

which are $\approx 2.5 \sigma$ smaller than the values predicted in the $\mathrm{SM}[18,19]$. Although the experimental confirmation of these results is still lacking and the further improvement is needed to increase the significance of the observed deviations w.r.t. the SM, the fact that the indications of LFUV do not concern only the tree-level decays but also those that are in the SM generated by quantum loops, stimulated a considerable activity in the flavor physics community. The observations that $R_{D^{(*)}}^{\exp }>R_{D^{(*)}}^{\mathrm{SM}}$ and $R_{K^{(*)}}^{\exp }<R_{K^{(*)}}^{\mathrm{SM}}$ are commonly referred to as the "B-physics anomalies". ${ }^{1}$

Apart from the mass effects, different phase space, and moderate hadronic uncertainties, no other reason can be found in the SM to explain the above-mentioned anomalies. In other words, in order to explain (or merely accommodate) the observed deviations w.r.t. $\mathrm{SM}$, one needs to invoke a scenario of physics beyond the SM. The simplest effective scenario is to introduce the couplings of left-handed fermions to new vector bosons. In practice that means that the New Physics (NP) effective operators will be of the " $(V-A) \times(V-A)$ " form, which are then fit with the measured $R_{K^{(*)}, D^{(*)}}^{\operatorname{epp}}$ values to reveal that the NP scale affecting the charged current processes is very different from the one needed to explain the

\footnotetext{
${ }^{1}$ For shortness, we only write $R_{D^{(*)}}^{\exp }>R_{D^{(*)}}^{\mathrm{SM}}$ and $R_{K^{(*)}}^{\exp }<R_{K^{(*)}}^{\mathrm{SM}}$, but one should also keep in mind that $R_{J / \psi}^{\exp }>R_{J / \psi}^{\mathrm{SM}}$.
} 
LFUV in the FCNC processes. In such a situation one needs to build a model in which one adjusts Yukawa couplings while keeping the NP scale the same for both types of $B$-physics anomalies. Another possibility is to build a model in which other Lorentz structures are also allowed (such as the scalar and/or tensor currents). One of the most popular scenarios in which most of these ideas can be tested are those based on the introduction of one or more leptoquark states, the colored new bosons which couple to both quarks and leptons. In this paper we are going to test the possibility of a single scalar or vector leptoquark (LQ) as a mediator of NP that can accommodate one or both of the $B$-physics anomalies, i.e. $R_{K^{(*)}}^{\exp }<R_{K^{(*)}}^{\mathrm{SM}}$ and/or $R_{D^{(*)}}^{\exp }>R_{D^{(*)}}^{\mathrm{SM}}$. We will go through various LQ scenarios to examine if any of them remains plausible. In doing so we will go for a minimalistic approach, i.e. that by extending the SM by one LQ involves the least number of new parameters, which then permits to test the model experimentally. In that respect, the current paper is similar to ref. [20] and could be viewed as its update. The new element is the fact that we combine the updated constraints arising from numerous low-energy physics observables with those deduced from the direct searches at the LHC. ${ }^{2}$ Since this kind of models can give rise to lepton flavor violation (LFV) [22], a particular attention will be devoted to the vector LQ model $\left(U_{1}\right)$ for which we will show that the improved experimental bounds on $\mathcal{B}\left(B_{s} \rightarrow \mu \tau\right)$ and/or $\mathcal{B}\left(B \rightarrow K^{(*)} \mu \tau\right)$, could validate or discard the model in its minimalistic form. Before we embark on the details of this work, we should emphasize that by a single LQ we mean one multiplet (singlet, doublet or triplet) of mass degenerate LQ's which carry the same quantum numbers of the SM gauge group.

This paper is organized as follows. In section 2, we remind the reader of a low-energy effective description of $b \rightarrow s \ell \ell$ and $b \rightarrow c \ell \bar{\nu}$ transitions, and we present the $1 \sigma$ bounds on the effective coefficients which accompany the hadronic matrix elements of dimension-six operators relevant to a general NP scenario. In section 3, we briefly go through the list of the single LQ solutions to the $B$-physics anomalies, and for each of them we compute the effective Wilson coefficients relevant to $R_{K^{(*)}}$ and to $R_{D^{(*)}}$, respectively. In section 4 , we discuss the bounds on the Yukawa couplings arising from the direct searches at LHC. Besides LQ pair production, we also comment on the measurements of tails of high- $p_{T}$ distributions of lepton pairs (in $p p \rightarrow \mu \mu, \tau \tau$ decays) which can be modified w.r.t. the SM by the $t$-channel LQ exchange. In section 5 the low-energy and high energy constraints are combined. We will then show that the only one that can accommodate both anomalies and survive all the constraints turns out to be the vector LQ $U_{1}$, the phenomenology of which is analyzed in more detail in section 6 , where we also show that the LFV processes $b \rightarrow s \mu \tau$, together with high- $p_{T}$ lepton tails at LHC, can provide complementary constraints and can be used to validate or invalidate model in its minimalistic form. We summarize our findings in section 7 .

\section{Effective theory description}

Effective theories provide an efficient way to describe the low-energy physics processes in which the short-distance physics is encoded in the so called Wilson coefficients, which

\footnotetext{
${ }^{2}$ Research on combining the low-energy constraints to building a SUSY inspired model with the results of direct searches at the LHC has been reported in ref. [21].
} 
are computed perturbatively, whereas the remaining long-distance physics is expressed in terms of a number of effective (dimension-six) operators, higher dimension operators being suppressed by powers of a high energy scale. In the SM the matching between the full and effective theories for the loop-induced $b \rightarrow s \ell^{+} \ell^{-}$process includes the next-to-nextto-leading logarithmic corrections and the resummation of potentially large logarithms has been made by means of the renormalization group equations [23]. Looking for NP in this context means to look for the non-SM contributions to the Wilson coefficients corresponding to the operators already present in the SM, in addition to those that are not present to the SM but which are allowed on the Lorentz symmetry grounds. In this section, for definiteness, we remind the reader of the low-energy effective theories relevant to both $b \rightarrow s \ell^{+} \ell^{-}$and $b \rightarrow c \ell \bar{\nu}$ decays.

\section{$2.1 \quad b \rightarrow s \ell_{1}^{-} \ell_{2}^{+}$and $R_{K^{(*)}}$}

Since we will be concerned with both lepton flavor conserving and LFV decay modes we will describe the effective Hamiltonian for a generic $b \rightarrow s \ell_{1}^{-} \ell_{2}^{+}$, with $\ell_{1,2} \in\{e, \mu, \tau\}$, which can be written as

$$
\begin{aligned}
\mathcal{H}_{\mathrm{eff}}=-\frac{4 G_{F}}{\sqrt{2}} V_{t b} V_{t s}^{*} & \left\{\sum_{i=1}^{6} C_{i}(\mu) \mathcal{O}_{i}(\mu)+\sum_{i=7,8}\left[C_{i}(\mu) \mathcal{O}_{i}(\mu)+\left(C_{i}(\mu)\right)^{\prime}\left(\mathcal{O}_{i}(\mu)\right)^{\prime}\right]\right. \\
& \left.+\sum_{i=9,10, S, P}\left[C_{i}^{\ell_{1} \ell_{2}}(\mu) \mathcal{O}_{i}^{\ell_{1} \ell_{2}}(\mu)+\left(C_{i}^{\ell_{1} \ell_{2}}(\mu)\right)^{\prime}\left(\mathcal{O}_{i}^{\ell_{1} \ell_{2}}(\mu)\right)^{\prime}\right]\right\}+ \text { h.c. }
\end{aligned}
$$

$C_{i}(\mu)$ and $C_{i}^{\ell_{1} \ell_{2}}(\mu)$ are the Wilson coefficients, while the effective operators relevant to our study are defined by

$$
\begin{aligned}
\mathcal{O}_{9}^{\ell_{1} \ell_{2}} & =\frac{e^{2}}{(4 \pi)^{2}}\left(\bar{s} \gamma_{\mu} P_{L} b\right)\left(\bar{\ell}_{1} \gamma^{\mu} \ell_{2}\right), & \mathcal{O}_{S}^{\ell_{1} \ell_{2}} & =\frac{e^{2}}{(4 \pi)^{2}}\left(\bar{s} P_{R} b\right)\left(\bar{\ell}_{1} \ell_{2}\right), \\
\mathcal{O}_{10}^{\ell_{1} \ell_{2}} & =\frac{e^{2}}{(4 \pi)^{2}}\left(\bar{s} \gamma_{\mu} P_{L} b\right)\left(\bar{\ell}_{1} \gamma^{\mu} \gamma^{5} \ell_{2}\right), & \mathcal{O}_{P}^{\ell_{1} \ell_{2}} & =\frac{e^{2}}{(4 \pi)^{2}}\left(\bar{s} P_{R} b\right)\left(\bar{\ell}_{1} \gamma^{5} \ell_{2}\right),
\end{aligned}
$$

in addition to the electromagnetic penguin operator, $\mathcal{O}_{7}=\left(e /(4 \pi)^{2}\right) m_{b}\left(\bar{s} \sigma_{\mu \nu} P_{R} b\right) F^{\mu \nu}$. The primed quantities in eq. (2.1) correspond to the chirality flipped operators, $\mathcal{O}_{i}^{\prime}$, which are obtained from $\mathcal{O}_{i}$ after replacing $P_{L} \leftrightarrow P_{R}$. From this Hamiltonian it is straightforward to compute the decay rates for $B_{s} \rightarrow \ell_{1} \ell_{2}$ and $B \rightarrow K^{(*)} \ell_{1} \ell_{2}$, cf. ref. [24]. In the following we will omit the dependence on the renormalization scale and take $C_{i} \equiv C_{i}\left(m_{b}\right)$.

By assuming that the NP couplings to electrons are negligible, it has been established that $R_{K}$ and $R_{K^{*}}$ can be explained by a purely vector Wilson coefficient, $C_{9}^{\mu \mu}<0$, or by a left-handed combination, $C_{9}^{\mu \mu}=-C_{10}^{\mu \mu}<0 .{ }^{3}$ The result of our fit, illustrated in figure 1 is

$$
C_{9}^{\mu \mu}=-C_{10}^{\mu \mu} \in(-0.85,-0.50)
$$

\footnotetext{
${ }^{3}$ An explanation of $R_{K^{(*)}}$ by NP couplings to electrons is disfavored by global analysis of the $b \rightarrow s \mu \mu$ observables, cf. refs. [25-28].
} 


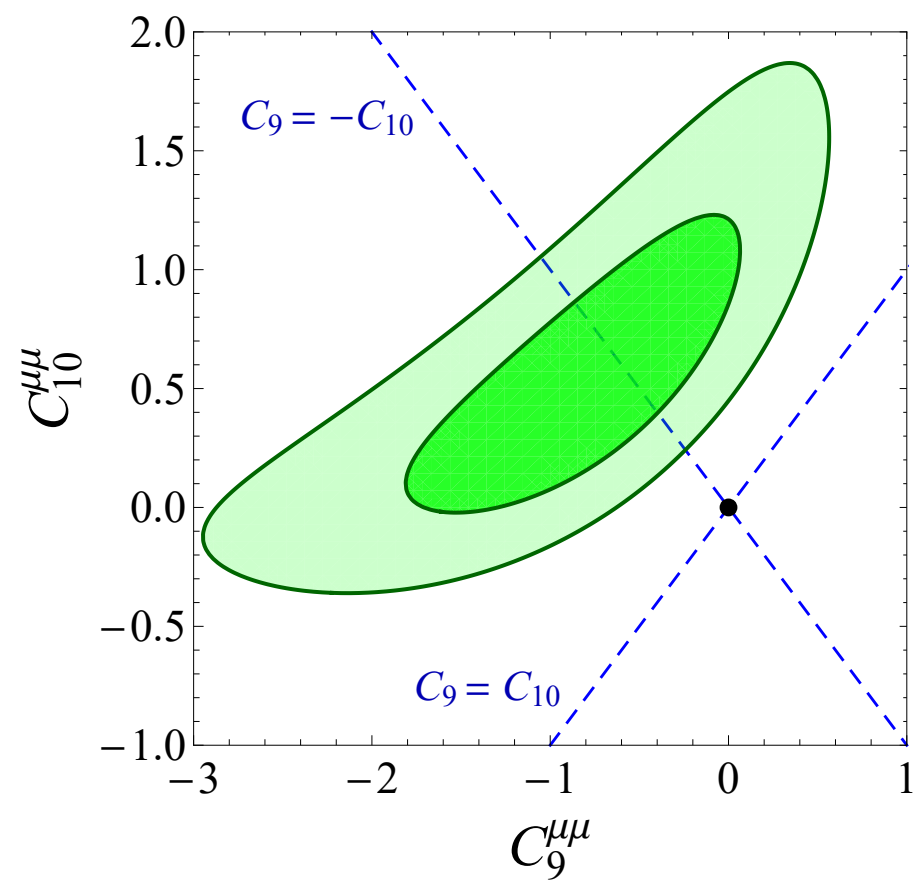

Figure 1. Low-energy fit to $R_{K}, R_{K^{*}}$ and $\mathcal{B}\left(B_{s} \rightarrow \mu \mu\right)$ in the plane $C_{9}^{\mu \mu}$ vs. $C_{10}^{\mu \mu}$. Darker (lighter) region is allowed to $1 \sigma(2 \sigma)$ accuracy. Blue dashed lines correspond to scenarios with $C_{9}^{\mu \mu}= \pm C_{10}^{\mu \mu}$, while the black dot denotes the SM point.

which deviates from the SM by almost $4 \sigma$. In this fit we used $R_{K^{(*)}}^{\exp }[16,17]$, and the theoretically clean $\mathcal{B}\left(B_{s} \rightarrow \mu \mu\right)^{\exp }=\left(3.0 \pm 0.6_{-0.2}^{+0.3}\right) \times 10^{-9}[29]^{4}$

The possibility of having $C_{9}^{\mu \mu}=-C_{10}^{\mu \mu}$ is particularly interesting because it is realized in several LQ scenarios [31]. From now on, for notational simplicity, we will omit the " $\mu \mu$ "-superscript.

\section{$2.2 \quad b \rightarrow c \ell \bar{\nu}$ and $R_{D^{(*)}}$}

The most general low-energy effective Lagrangian involving all of the dimension-six operators capable to generate a (semi-)leptonic decay via charged currents reads

$$
\begin{aligned}
\mathcal{L}_{\text {eff }}=-2 \sqrt{2} G_{F} V_{u d} & {\left[\left(1+g_{V_{L}}\right)\left(\bar{u}_{L} \gamma_{\mu} d_{L}\right)\left(\bar{\ell}_{L} \gamma^{\mu} \nu_{L}\right)+g_{V_{R}}\left(\bar{u}_{R} \gamma_{\mu} d_{R}\right)\left(\bar{\ell}_{L} \gamma^{\mu} \nu_{L}\right)\right.} \\
& +g_{S_{L}}(\mu)\left(\bar{u}_{R} d_{L}\right)\left(\bar{\ell}_{R} \nu_{L}\right)+g_{S_{R}}(\mu)\left(\bar{u}_{L} d_{R}\right)\left(\bar{\ell}_{R} \nu_{L}\right) \\
& \left.+g_{T}(\mu)\left(\bar{u}_{R} \sigma_{\mu \nu} d_{L}\right)\left(\bar{\ell}_{R} \sigma^{\mu \nu} \nu_{L}\right)\right]+ \text { h.c. }
\end{aligned}
$$

where $u$ and $d$ stand for a generic up- and down-type quarks, and $g_{i} \equiv g_{i}\left(m_{b}\right)$ are the effective NP couplings with $i \in\left\{V_{L(R)}, S_{L(R)}, T\right\}$. In order to describe the anomalies observed in the exclusive $b \rightarrow c \ell \bar{\nu}$ decays one necessarily needs to introduce the new bosonic fields above the electroweak scale. Such an extended theory should also respect the $\mathrm{SU}(2)_{L} \times \mathrm{U}(1)_{Y}$ symmetry which means that $g_{V_{R}}$ should be lepton flavor universal

\footnotetext{
${ }^{4}$ Notice that the measured $\mathcal{B}\left(B_{s} \rightarrow \mu \mu\right)^{\text {exp }}$, agrees with the $\mathrm{SM}$ value $\mathcal{B}\left(B_{s} \rightarrow \mu \mu\right)^{\mathrm{SM}}=(3.65 \pm 0.23) \times$ $10^{-9}[30]$.
} 
at dimension-six, and as such it is irrelevant for the discussion that follows [32, 33]. In other words, we are left with four effective coefficients, $g_{V_{L}}, g_{S_{L}}, g_{S_{R}}$ and $g_{T}$ which can potentially contribute to $R_{D^{(*)}}$.

To determine the allowed values of the effective couplings $g_{i}$ we assume that NP only contributes to the transition $b \rightarrow c \tau \bar{\nu}$, and that its effect is negligible to the electron and muon modes. ${ }^{5}$ We use the $B \rightarrow D$ semileptonic form factors computed by means of lattice QCD in refs. $[9,10]$. Since the $B \rightarrow D^{*}$ form factors at non-zero recoil are still not available from LQCD, we consider the ones extracted from the measured angular distribution of $B \rightarrow D^{*}(\rightarrow D \pi) l \bar{\nu}(l \in\{e, \mu\})$ given in ref. [8], and combine them with the ratios $A_{0}\left(q^{2}\right) / A_{1}\left(q^{2}\right)$ and $T_{1-3}\left(q^{2}\right) / A_{1}\left(q^{2}\right)$ computed in ref. [14]. By using these theoretical inputs and the experimental values given in eq. (1.2) we were able to constrain the values of effective coefficients in eq. (2.4) and thus accommodate $R_{D^{(*)}}$. The first solution we consider is the coefficient $g_{V_{L}}>0$, which corresponds to an overall rescaling of the SM. The allowed $1 \sigma$ range in this case reads ${ }^{6}$

$$
\left.g_{V_{L}}\right|_{b \rightarrow c \tau \nu_{\tau}} \in(0.09,0.13) .
$$

Other solutions involving the coefficients $g_{S_{L}}$ and/or $g_{T}$ have also been considered in the literature [34-44]. In particular, two specific scalar LQ scenarios predict $g_{S_{L}}= \pm 4 g_{T}$ at the scale $\mu=m_{\mathrm{LQ}}$, which is of the order of $1 \mathrm{TeV}$ [31]. That relation is modified when running down to $\mu=m_{b}$. After including in the renormalization group running the oneloop electroweak corrections, in addition to the three-loop QCD anomalous dimensions, the relations $g_{S_{L}}=+4 g_{T}$ and $g_{S_{L}}=-4 g_{T}$ become $g_{S_{L}} \approx+8.14 g_{T}$ and $g_{S_{L}} \approx-8.5 g_{T}$ at $\mu=m_{b}$, respectively [45]. The low-energy fits to various combinations of effective coefficients are shown in figure 2. In the same plot, we superimpose the limits derived from the $B_{c}$-meson lifetime, which is particularly efficient to constraint the pseudoscalar contribution [46, 47]. More precisely, we consider the conservative limit $\mathcal{B}\left(B_{c} \rightarrow \tau \bar{\nu}\right) \lesssim 30 \%$ and the expression

$$
\mathcal{B}\left(B_{c} \rightarrow \tau \bar{\nu}\right)=\tau_{B_{c}} \frac{m_{B_{c}} f_{B_{c}}^{2} G_{F}^{2}\left|V_{c b}\right|^{2}}{8 \pi} m_{\tau}^{2}\left(1-\frac{m_{\tau}^{2}}{m_{B_{c}}^{2}}\right)^{2}\left|1+g_{V_{L}}+\frac{\left(g_{S_{R}}-g_{S_{L}}\right) m_{B_{c}}^{2}}{m_{\tau}\left(m_{b}+m_{c}\right)}\right|^{2}
$$

from which we derive that

$$
g_{P}\left(m_{b}\right) \equiv g_{S_{R}}\left(m_{b}\right)-g_{S_{L}}\left(m_{b}\right) \in(-1.14,0.68) .
$$

By combining this constraint with the low-energy fit to $R_{D^{(*)}}$ described above, we conclude in figure 2 that not only the scenario with $g_{V_{L}}>0$ can accommodate $R_{D^{(*)}}$, but also other scenarios such as $g_{T}\left(m_{b}\right) \neq 0, g_{S_{L}}=-4 g_{T}>0$. Furthermore, plausible solutions are obtained for $g_{S_{L}}= \pm 4 g_{T}$ but allowing the couplings to be mostly imaginary. These

\footnotetext{
${ }^{5}$ That assumption is a very good approximation to the realistic situation. As we shall see, we find that the couplings of leptoquarks to $b$ and $\tau$ are indeed much larger than those involving muons so that the physics discussion of $R_{D^{(*)}}$ remains unchanged after setting the couplings to muons to zero.

${ }^{6} \mathrm{We}$ disregard the solution with large and negative values of $g_{V_{L}}$, since this possibility would require excessively large NP couplings.
} 

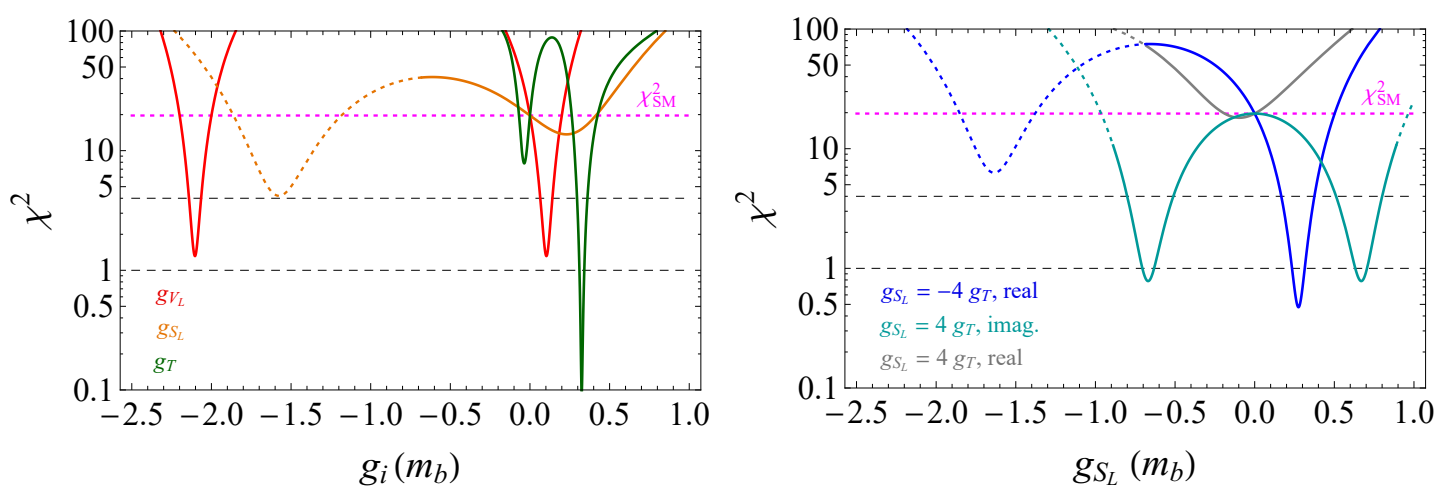

Figure 2. $\chi^{2}$ values for individual effective coefficients fits of $R_{D}$ and $R_{D^{*}}$, compared to the SM value, $\chi_{\mathrm{SM}}^{2} \approx 19.7$. In the left panel, $\chi^{2}$ is plotted against $g_{V_{L}}, g_{S_{L}}$ and $g_{T}$ at $\mu=m_{b}$. In the right panel, $\chi^{2}$ is plotted against $g_{S_{L}}\left(m_{b}\right)$ by assuming $g_{S_{L}}= \pm 4 g_{T}$ at $\mu=1 \mathrm{TeV}$, for purely imaginary and real couplings. The dashed regions correspond to the values excluded by the $B_{c^{-}}$ lifetime constraints, see text for details.

findings are in agreement with the literature, cf. ref. [34], which are updated in this paper with the most recent experimental and theoretical inputs and extended by allowing the possibility of imaginary couplings.

\section{Leptoquark models for $\boldsymbol{R}_{\boldsymbol{K}^{(*)}}$ and/or $\boldsymbol{R}_{\boldsymbol{D}^{(*)}}$}

In this section we briefly review the LQ models proposed to accommodate the $B$-physics anomalies by introducing a single mediator. We adopt the notation of ref. [31] and specify the LQ by their SM quantum numbers, $\left(\mathrm{SU}(3)_{c}, \mathrm{SU}(2)_{L}\right)_{Y}$, where the electric charge, $Q=$ $Y+T_{3}$, is the sum of the hypercharge $(Y)$ and the third weak isospin component $\left(T_{3}\right)$. In the left-handed doublets, $Q_{i}=\left[\left(V^{\dagger} u_{L}\right)_{i} d_{L i}\right]^{T}$ and $L_{i}=\left[\left(U \nu_{L}\right)_{i} \ell_{L i}\right]^{T}$, the matrices $V$ and $U$ are the CKM and Pontecorvo-Maki-Nakagawa-Sakata (PMNS) matrices, respectively. Since the neutrino masses are insignificant for the phenomenology we study in this paper, we can set $U=\mathbb{1}$.

\subsection{Scalar leptoquarks}

\subsection{1 $S_{3}=(\overline{3}, 3)_{1 / 3}$}

The first scenario we consider is with $S_{3}=(\overline{\mathbf{3}}, \mathbf{3})_{1 / 3}$, a weak triplet of scalar LQ states with hypercharge $1 / 3 .{ }^{7}$ Remarkably, $S_{3}$ is the only scalar particle that can simultaneously account for $R_{K}^{\exp }<R_{K}^{\mathrm{SM}}$ and $R_{K^{*}}^{\exp }<R_{K^{*}}^{\mathrm{SM}}$ at tree-level [48-51]. The Yukawa Lagrangian of $S_{3}$ reads [31]

$$
\mathcal{L}_{S_{3}}=y_{L}^{i j} \overline{Q_{i}^{C}} i \tau_{2}\left(\tau_{k} S_{3}^{k}\right) L_{j}+\text { h.c. },
$$

where $\tau_{k}$ is one of the Pauli matrices $(k=1,2,3), S_{3}^{k}$ is a component of the LQ triplet, and $y_{L}$ stands for a generic Yukawa matrix. We have neglected the LQ couplings to diquarks

\footnotetext{
${ }^{7}$ We follow a common practice and in this paper we consider the LQ's belonging to the same multiplet to be mass degenerate.
} 
in eq. (3.1) in order to ensure the proton stability [31]. Otherwise one should devise an appropriate symmetry to suppress these couplings that are known to be tightly constrained by experimental limits on the proton lifetime. ${ }^{8}$ It is convenient to rewrite eq. (3.1) in terms of charge eigenstates as:

$$
\begin{aligned}
\mathcal{L}_{S_{3}}= & -y_{L}^{i j} \overline{d_{L i}^{C}} \nu_{L j} S_{3}^{(1 / 3)}-\sqrt{2} y_{L}^{i j} \overline{d_{L i}^{C}} \ell_{L j} S_{3}^{(4 / 3)} \\
& +\sqrt{2}\left(V^{*} y_{L}\right)_{i j} \overline{u_{L i}^{C}} \nu_{L j} S_{3}^{(-2 / 3)}-\left(V^{*} y_{L}\right)_{i j} \overline{u_{L i}^{C}} \ell_{L j} S_{3}^{(1 / 3)}+\text { h.c. },
\end{aligned}
$$

from which one can easily extract the Wilson coefficients for the $b \rightarrow s \ell_{l}^{-} \ell_{k}^{+}$decay, namely,

$$
C_{9}^{k l}=-C_{10}^{k l}=\frac{\pi v^{2}}{V_{t b} V_{t s}^{*} \alpha_{\mathrm{em}}} \frac{y_{L}^{b k}\left(y_{L}^{s l}\right)^{*}}{m_{S_{3}}^{2}},
$$

which is precisely the effective scenario needed to accommodate $R_{K^{(*)}}^{\exp }<R_{K^{(*)}}^{\mathrm{SM}}$. Notice once again that in this discussion $C_{9,10}^{k l}$ refer only to the LQ contribution, that is to be added to the SM value which is non-zero in the lepton flavor conserving case $(l=k)$. Similarly, we also read off the contribution arising from this LQ to the $b \rightarrow c \ell \bar{\nu}_{\ell^{\prime}}$ transition and find

$$
g_{V_{L}}=-\frac{v^{2} y_{L}^{b \ell^{\prime}}\left(V y_{L}^{*}\right)_{c \ell}}{4 V_{c b} m_{S_{3}}^{2}}=-\frac{v^{2}}{4 m_{S_{3}}^{2}} y_{L}^{b \ell^{\prime}}\left[\left(y_{L}^{b \ell}\right)^{*}+\frac{V_{c s}}{V_{c b}}\left(y_{L}^{s \ell}\right)^{*}+\frac{V_{c d}}{V_{c b}}\left(y_{L}^{d \ell}\right)^{*}\right] .
$$

We see that the coupling $y_{L}^{b \ell}$ gives a negative contribution to $g_{V_{L}}$, clearly at odds with $R_{D^{(*)}}^{\exp }>R_{D^{(*)}}^{\mathrm{SM}}$. Note that the terms depending on $y_{L}^{s \ell}$ and $y_{L}^{d \ell}$ are not necessarily negative, but they are tightly constrained by other flavor limits, such as $\mathcal{B}(B \rightarrow K \bar{\nu} \nu)$ and the frequency of oscillations in the $B_{d, s}^{0}-\bar{B}_{d, s}^{0}$ system $\left(\Delta m_{B_{d, s}}\right)$, so that this scenario cannot accommodate $R_{D^{(*)}}$.

\subsection{2 $\quad R_{2}=(3,2)_{7 / 6}$}

The second scenario we consider is $R_{2}=(\mathbf{3}, \mathbf{2})_{7 / 6}$, the weak doublet of scalar LQ's with hypercharge $Y=7 / 6$. This scenario is known to be unsatisfactory at tree level because it leads to $R_{K^{(*)}}>R_{K^{(*)}}^{\mathrm{SM}}$, clearly disfavored by the data. One can get around this problem, as proposed in ref. [52], and generate corrections to $R_{K^{(*)}}$ at loop-level and accommodate $R_{K^{(*)}}^{\exp }<R_{K^{(*)}}^{\mathrm{SM}}$. Interestingly, $R_{2}$ is the only scalar LQ for which the proton stability is automatically preserved [53]. The most general Lagrangian describing the Yukawa interactions with $R_{2}$ can be written as,

$$
\mathcal{L}_{R_{2}}=y_{R}^{i j} \bar{Q}_{i} \ell_{R j} R_{2}-y_{L}^{i j} \bar{u}_{R i} R_{2} i \tau_{2} L_{j}+\text { h.c. },
$$

where $y_{L}$ and $y_{R}$ are the Yukawa matrices, and $\mathrm{SU}(2)_{L}$ indices have been omitted for simplicity. More explicitly, in terms of the electric charge eigenstates, the Lagrangian (3.5) can be written as

$$
\begin{aligned}
\mathcal{L}_{R_{2}}= & \left(V y_{R}\right)_{i j} \bar{u}_{L i} \ell_{R j} R_{2}^{(5 / 3)}+\left(y_{R}\right)_{i j} \bar{d}_{L i} \ell_{R j} R_{2}^{(2 / 3)} \\
& +\left(y_{L}\right)_{i j} \bar{u}_{R i} \nu_{L j} R_{2}^{(2 / 3)}-\left(y_{L}\right)_{i j} \bar{u}_{R i} \ell_{L j} R_{2}^{(5 / 3)}+\text { h.c. }
\end{aligned}
$$

\footnotetext{
${ }^{8}$ See ref. [48] for illustration of a Grand Unification scenario in which these couplings are absent in a concrete SU(5) set-up.
} 
As mentioned above, the tree-level contribution to the Wilson coefficients amounts to

$$
C_{9}^{k l}=C_{10}^{k l} \stackrel{\text { tree }}{=}-\frac{\pi v^{2}}{2 V_{t b} V_{t s}^{*} \alpha_{\mathrm{em}}} \frac{y_{R}^{s l}\left(y_{R}^{b k}\right)^{*}}{m_{R_{2}}^{2}},
$$

which leads to $R_{K^{(*)}}>R_{K^{(*)}}^{\mathrm{SM}}$, in conflict with experiment. Instead, if the LQ corrections start at loop-level, which can be achieved by setting $y_{R} \equiv 0$, one finds [52]

$$
C_{9}^{k l}=-C_{10}^{k l} \stackrel{\text { loop }}{=} \sum_{u, u^{\prime} \in\{u, c, t\}} \frac{V_{u b} V_{u^{\prime} s}^{*}}{V_{t b} V_{t s}^{*}} y_{L}^{u^{\prime} k}\left(y_{L}^{u l}\right)^{*} \mathcal{F}\left(x_{u}, x_{u^{\prime}}\right),
$$

where $x_{i}=m_{i}^{2} / m_{W}^{2}$, and the loop function reads,

$$
\begin{aligned}
\mathcal{F}\left(x_{u}, x_{u^{\prime}}\right)=\frac{\sqrt{x_{u} x_{u^{\prime}}}}{32 \pi \alpha_{\mathrm{em}}} & {\left[\frac{x_{u^{\prime}}\left(x_{u^{\prime}}-4\right) \log x_{u^{\prime}}}{\left(x_{u^{\prime}}-1\right)\left(x_{u}-x_{u^{\prime}}\right)\left(x_{u^{\prime}}-x_{\Delta}\right)}+\frac{x_{u}\left(x_{u}-4\right) \log x_{u}}{\left(x_{u}-1\right)\left(x_{u^{\prime}}-x_{u}\right)\left(x_{u}-x_{\Delta}\right)}\right.} \\
& \left.-\frac{x_{\Delta}\left(x_{\Delta}-4\right) \log x_{\Delta}}{\left(x_{\Delta}-1\right)\left(x_{\Delta}-x_{u}\right)\left(x_{\Delta}-x_{u^{\prime}}\right)}\right] .
\end{aligned}
$$

Furthermore, this LQ state contributes to the transition $b \rightarrow c \tau \bar{\nu}_{\ell^{\prime}}$ via the following coefficients,

$$
g_{S_{L}}=4 g_{T}=\frac{v^{2}}{4 V_{c b}} \frac{y_{L}^{c \ell^{\prime}}\left(y_{R}^{b \ell}\right)^{*}}{m_{R_{2}}^{2}},
$$

obtained by tree-level matching at the scale $\mu=m_{R_{2}}$. This scenario can accommodate the observed deviations in $R_{D^{(*)}}$ for complex and even purely imaginary couplings, as it can be seen in figure 2 , and in refs. [35, 54, 55].

A simultaneous explanation of $R_{K^{(*)}}$ and $R_{D^{(*)}}$ is, however, excluded due to the stringent limits on $\mathcal{B}(\tau \rightarrow \mu \gamma)$, which would receive a contribution enhanced by a factor of $m_{t} / m_{\tau}$ at the amplitude level, as discussed in ref. [52]. Therefore, this scenario can accommodate either $R_{K^{(*)}}$ or $R_{D^{(*)}}$, but not both.

\subsection{3 $\widetilde{R}_{2}=(3,2)_{1 / 6}$}

Another important scenario to consider is $\widetilde{R}_{2}=(\mathbf{3}, \mathbf{2})_{1 / 6}$, the weak doublet of scalar LQ's with hypercharge $Y=1 / 6$, which couples to SM fermions through a single gauge invariant operator, namely, [56]

$$
\begin{aligned}
\mathcal{L}_{\widetilde{R}_{2}} & =-y_{L}^{i j} \overline{d_{R i}} \widetilde{R}_{2} i \tau_{2} L_{j}+\text { h.c. } \\
& =-y_{L}^{i j} \overline{d_{R i}} \ell_{L j} \widetilde{R}_{2}^{(2 / 3)}+y_{L}^{i j} \overline{d_{R i}} \nu_{L j} \widetilde{R}_{2}^{(-1 / 3)}+\text { h.c. },
\end{aligned}
$$

where $y_{L}$ is a generic matrix of Yukawa couplings. Another appealing feature of this scenario is that, like the $R_{2}$ model, the potentially troublesome diquark couplings to LQ are absent. Proton decay can still be generated in this scenario but by higher order operators which can be eliminated by imposing a suitable symmetry in a way similar to what has been done, for example, in ref. [57]. As before, we again identify the Wilson coefficients arising from the tree level contributions to $b \rightarrow s \ell_{l}^{-} \ell_{k}^{+}$in this model and find,

$$
C_{9}^{\prime k l}=-C_{10}^{\prime k l}=-\frac{\pi v^{2}}{2 V_{t b} V_{t s}^{*} \alpha_{\mathrm{em}}} \frac{y_{L}^{s k}\left(y_{L}^{b l}\right)^{*}}{m_{\widetilde{R}_{2}}^{2}},
$$


which turned out to be in agreement with $R_{K}^{\exp }<R_{K}^{\mathrm{SM}}$ but in conflict with $R_{K^{*}}^{\exp }<R_{K^{*}}^{\mathrm{SM}}$, cf. discussion in ref. [56]. Furthermore, the Yukawa interactions in eq. (3.11) do not contribute to the charged current processes, such as the transition $b \rightarrow c \tau \bar{\nu}$. This limitation can be overcome by introducing light right-handed neutrinos to this set-up [58]. In that case, scalar and tensor operators will be generated through the gauge invariant operator $\bar{Q} \widetilde{R}_{2} \nu_{R}$, which will not interfere with the SM contributions and therefore can provide only a small shift with respect to the SM predictions.

\subsection{4 $S_{1}=(\overline{3}, 1)_{1 / 3}$}

Finally, one can also consider a scenario with $S_{1}=(\overline{\mathbf{3}}, \mathbf{1})_{1 / 3}$, a weak singlet scalar LQ with hypercharge $Y=1 / 3$. This model was deemed to be viable in ref. [54] for accommodating $R_{D^{(*)}}^{\exp }>R_{D^{(*)}}^{\mathrm{SM}}$. The most general Yukawa Lagrangian of $S_{1}$ reads

$$
\begin{aligned}
\mathcal{L}_{S_{1}} & =y_{L}^{i j} \overline{Q^{C}} i \tau_{2} L_{j} S_{1}+y_{R}^{i j} \overline{u_{R i}^{C}} e_{R j} S_{1}+\text { h.c. } \\
& =S_{1}\left[\left(V^{*} y_{L}\right)_{i j} \overline{u_{L i}^{C}} \ell_{L j}-y_{L}^{i j} \overline{d_{L i}^{C}} \nu_{L j}+y_{R}^{i j} \overline{u_{R i}^{C}} \ell_{R j}\right]+\text { h.c. },
\end{aligned}
$$

where $y_{L}$ and $y_{R}$ are generic Yukawa matrices. Like in the case of $S_{3}$ we omitted the terms involving diquark couplings to LQ, which must be forbidden by a symmetry to protect the proton stability. By integrating out the LQ state we obtain that, at the matching scale $\mu=m_{S_{1}}$,

$$
\begin{aligned}
& g_{V_{L}}=\frac{v^{2}}{4 V_{c b}} \frac{y_{L}^{b \ell^{\prime}}\left(V y_{L}^{*}\right)_{c \ell}}{m_{S_{1}}^{2}}, \\
& g_{S_{L}}=-4 g_{T}=-\frac{v^{2}}{4 V_{c b}} \frac{y_{L}^{b \ell^{\prime}}\left(y_{R}^{c \ell}\right)^{*}}{m_{S_{1}}^{2}} .
\end{aligned}
$$

Although this LQ does not contribute to the transition $b \rightarrow s \ell_{l}^{-} \ell_{k}^{+}$at tree-level, the effective coefficients $C_{9(10)}$ receive contributions at loop-level, namely [59]

$$
\begin{aligned}
C_{9}^{k l}+C_{10}^{k l}= & \frac{m_{t}^{2}}{8 \pi \alpha_{\mathrm{em}} m_{S_{1}}^{2}}\left(V^{*} y_{L}\right)_{t k}\left(V^{*} y_{L}\right)_{t l}^{*}-\frac{v^{2}}{32 \pi \alpha_{\mathrm{em}} m_{S_{1}}^{2}} \frac{\left(y_{L} \cdot y_{L}^{\dagger}\right)_{b s}}{V_{t b} V_{t s}^{*}}\left(y_{L}^{\dagger} \cdot y_{L}\right)_{k l}, \\
C_{9}^{k l}-C_{10}^{k l}= & \frac{m_{t}^{2}}{8 \pi \alpha_{\mathrm{em}} m_{S_{1}}^{2}}\left(y_{R}\right)_{t k}\left(y_{R}\right)_{t l}^{*}\left[\log \frac{m_{S_{1}}^{2}}{m_{t}^{2}}-f\left(x_{t}\right)\right] \\
& -\frac{v^{2}}{32 \pi \alpha_{\mathrm{em}} m_{S_{1}}^{2}} \frac{\left(y_{L} \cdot y_{L}^{\dagger}\right)_{b s}}{V_{t b} V_{t s}^{*}}\left(y_{L}^{\dagger} \cdot y_{L}\right)_{k l},
\end{aligned}
$$

where $x_{t}=m_{t}^{2} / m_{W}^{2}$ and $f\left(x_{t}\right)=1+\frac{3}{x_{t}-1}\left(\frac{\log x_{t}}{x_{t}-1}-1\right)$. The possibility of explaining $R_{K^{(*)}}$ and/or $R_{D^{(*)}}$ in this scenario will be discussed in section 5 .

\section{$3.2 \quad$ Vector leptoquarks}

\subsection{1 $U_{1}=(3,1)_{2 / 3}$}

The first scenario of this sort we consider is $U_{1}=(\overline{\mathbf{3}}, \mathbf{1})_{2 / 3}$, the weak singlet vector LQ, which received considerable attention because it can provide a simultaneous explanation 
to the anomalies in $b \rightarrow s$ and $b \rightarrow c$ transitions [60]. The most general Lagrangian consistent with the SM gauge symmetry allows couplings to both left-handed and righthanded fermions, namely,

$$
\mathcal{L}_{U_{1}}=x_{L}^{i j} \bar{Q}_{i} \gamma_{\mu} U_{1}^{\mu} L_{j}+x_{R}^{i j} \bar{d}_{R i} \gamma_{\mu} U_{1}^{\mu} \ell_{R j}+\text { h.c. },
$$

where $x_{L}^{i j}$ and $x_{R}^{i j}$ are the couplings. The contribution of the left-handed couplings to the effective Lagrangian (2.1) amounts to

$$
C_{9}^{k l}=-C_{10}^{k l}=-\frac{\pi v^{2}}{V_{t b} V_{t s}^{*} \alpha_{\mathrm{em}}} \frac{x_{L}^{s l}\left(x_{L}^{b k}\right)^{*}}{m_{U_{1}}^{2}},
$$

as required by the observation of $R_{K^{(*)}}^{\exp }<R_{K^{(*)}}^{\mathrm{SM}}$. Switching on the right-handed couplings, $x_{R} \neq 0$, amounts to contributions to other Wilson coefficients, $C_{9}^{\prime}=C_{10}^{\prime}, C_{S}=-C_{P}$ and $\left(C_{S}\right)^{\prime}=\left(C_{P}\right)^{\prime}$. However, since the latter Wilson coefficients are disfavored by the current $b \rightarrow s$ data, we will set the right-handed couplings to zero and call such a scenario the minimal $U_{1}$ model. Furthermore, this scenario also contributes to $b \rightarrow c \ell \bar{\nu}_{\ell^{\prime}}$ by giving rise to the effective coefficient

$$
\begin{aligned}
g_{V_{L}} & =\frac{v^{2}\left(V x_{L}\right)_{c \ell^{\prime}}\left(x_{L}^{b \ell}\right)^{*}}{2 V_{c b} m_{U_{1}}^{2}} \\
& =\frac{v^{2}}{2 m_{U_{1}}^{2}}\left(x_{L}^{b \ell}\right)^{*}\left[x_{L}^{b \ell^{\prime}}+\frac{V_{c s}}{V_{c b}} x_{L}^{s \ell^{\prime}}+\frac{V_{c d}}{V_{c b}} x_{L}^{d \ell^{\prime}}\right],
\end{aligned}
$$

which is clearly acceptable since the leading term implies $g_{V_{L}}>0$, in agreement with the observed $R_{D^{(*)}}^{\exp }>R_{D^{(*)}}^{\mathrm{SM}}$.

A peculiarity of this scenario is the absence of contributions to the transition $b \rightarrow$ $s \nu \bar{\nu}[20,60]$. Importantly, however, this model is nonrenormalizable, which undermines its predictivity at the loop-level unless the ultraviolet (UV) mechanism generating the $U_{1}$ mass is explicitly specified, see refs. [53, 61-66] for concrete examples.

\subsection{2 $U_{3}=(3,3)_{2 / 3}$}

The last scenario we consider is the weak triplet of vector LQ's, $U_{3}=(\mathbf{3}, \mathbf{3})_{2 / 3}$ [67]. Due to gauge symmetry, this LQ couples only to the left-handed SM fermion doublets, with the most general Lagrangian being [31]

$$
\mathcal{L}_{U_{3}}=x_{L}^{i j} \bar{Q}_{i} \gamma^{\mu}\left(\tau_{k} U_{3 \mu}^{k}\right) L_{j}+\text { h.c. }
$$

Using eq. (3.20) and matching at tree-level onto the effective Lagrangian in eq. (2.1), we obtain that

$$
C_{9}^{k l}=-C_{10}^{k l}=-\frac{\pi v^{2}}{V_{t b} V_{t s}^{*} \alpha_{\mathrm{em}}} \frac{x_{L}^{s l}\left(x_{L}^{b k}\right)^{*}}{m_{U_{3}}^{2}},
$$

which can accommodate $R_{K^{(*)}}$, in a way similar to the $U_{1}$ model. Concerning the effective operators in the charged-current case (2.4), the only non-vanishing effective coefficient is 


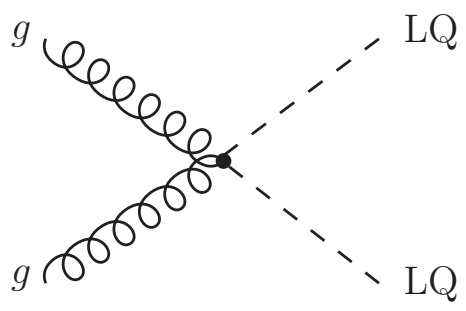

(a)

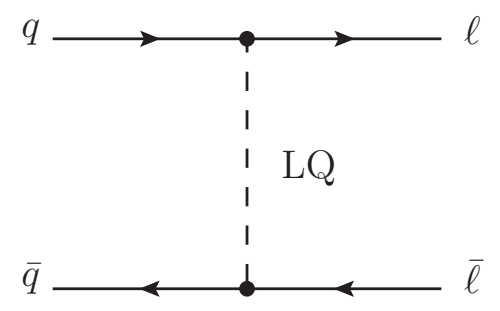

(b)

Figure 3. (a) Representative Feynman diagram for LQ pair production via QCD interactions. (b) Feynman diagram for LQ $t$-channel exchange in $p p \rightarrow \ell \bar{\ell}$ production at the LHC. The dashed propagator represents either a scalar or vector LQ state.

again $g_{V_{L}}$ which is given by

$$
\begin{aligned}
g_{V_{L}} & =-\frac{v^{2}\left(V x_{L}\right)_{c \ell^{\prime}}\left(x_{L}^{b \ell}\right)^{*}}{2 V_{c b} m_{U_{3}}^{2}} \\
& =-\frac{v^{2}}{2 m_{U_{3}}^{2}}\left(x_{L}^{b \ell}\right)^{*}\left[x_{L}^{b \ell^{\prime}}+\frac{V_{c s}}{V_{c b}} x_{L}^{s \ell^{\prime}}+\frac{V_{c d}}{V_{c b}} x_{L}^{d \ell^{\prime}}\right] .
\end{aligned}
$$

We see that, similar to the scenario with $S_{3}$, this model cannot accommodate the deviation in $R_{D^{(*)}}$ because the term proportional to $\left|x_{L}^{b \tau}\right|^{2}$ is negative, while the others are tightly constrained by other flavor physics observables, such as $\mathcal{B}(B \rightarrow K \nu \bar{\nu})$.

Similarly to $U_{1}$, the $U_{3}$ model is generally nonrenormalizable. Nevertheless, under certain circumstances, loops involving $U_{3}$ can be calculated. More precisely, if the $3 \times 3$ matrix $x_{L}$ from eq. (3.20) is unitary, UV-divergences appearing in loop-induced FCNCs mediated by $U_{3}$ are canceled through the GIM mechanism. However, the price to pay for having a unitary coupling matrix is that LQ couplings to first generation SM fermions, such as $e, d$, or $u$, can no longer be avoided. In turn, the presence of such couplings is in strong conflict with LFV bounds from $\mu-e$ conversion in Au nuclei and from $\mathcal{B}\left(K_{L} \rightarrow \mu e\right)$, which exclude the $U_{3}$ scenario with unitary $x_{L}$ as a viable explanation of the $b \rightarrow s$ anomalies, see discussion in ref. [20].

\section{High- $p_{T}$ phenomenology}

\subsection{Direct limits on pair-produced LQs}

An efficient way to set limits on LQs is to directly search for them at hadron colliders. At the LHC one of the most significant example of such a processes is the pair production $g g(q \bar{q}) \rightarrow \mathrm{LQ}^{\dagger} \mathrm{LQ}$, shown in figure $3(\mathrm{a})$. In both ATLAS and CMS the searches for this process in different decay channels into second and/or third generation quarks and leptons, $\mathrm{LQ}^{\dagger} \mathrm{LQ} \rightarrow q \bar{q} \ell \bar{\ell}, q \bar{q} \nu \bar{\nu}$, have been made. The results of these searches lead to model independent bounds on both the mass and branching fractions of the LQ.

In table 1 we list the most recent lower limits on the masses of second/third generation scalar and vector LQs relevant to this work, for benchmark branching ratios set to 


\begin{tabular}{|c|c|c|c|c|}
\hline Decays & LQs & Scalar LQ limits & Vector LQ limits & $\mathcal{L}_{\text {int }} /$ Ref. \\
\hline$j j \tau \bar{\tau}$ & $S_{1}, R_{2}, S_{3}, U_{1}, U_{3}$ & - & - & - \\
\hline$b \bar{b} \tau \bar{\tau}$ & $R_{2}, S_{3}, U_{1}, U_{3}$ & $850(550) \mathrm{GeV}$ & $1550(1290) \mathrm{GeV}$ & $12.9 \mathrm{fb}^{-1} \quad[69]$ \\
\hline$t \bar{t} \tau \bar{\tau}$ & $S_{1}, R_{2}, S_{3}, U_{3}$ & $900(560) \mathrm{GeV}$ & $1440(1220) \mathrm{GeV}$ & $35.9 \mathrm{fb}^{-1} \quad[70]$ \\
\hline$j j \mu \bar{\mu}$ & $S_{1}, R_{2}, S_{3}, U_{1}, U_{3}$ & $1530(1275) \mathrm{GeV}$ & $2110(1860) \mathrm{GeV}$ & $35.9 \mathrm{fb}^{-1} \quad[71]$ \\
\hline$b \bar{b} \mu \bar{\mu}$ & $R_{2}, U_{1}, U_{3}$ & $1400(1160) \mathrm{GeV}$ & $1900(1700) \mathrm{GeV}$ & $36.1 \mathrm{fb}^{-1} \quad[72]$ \\
\hline$t \bar{t} \mu \bar{\mu}$ & $S_{1}, R_{2}, S_{3}, U_{3}$ & $1420(950) \mathrm{GeV}$ & $1780(1560) \mathrm{GeV}$ & $36.1 \mathrm{fb}^{-1}[73,74]$ \\
\hline$j j \nu \bar{\nu}$ & $R_{2}, S_{3}, U_{1}, U_{3}$ & $980(640) \mathrm{GeV}$ & $1790(1500) \mathrm{GeV}$ & $35.9 \mathrm{fb}^{-1}[75]$ \\
\hline$b \bar{b} \nu \bar{\nu}$ & $S_{1}, R_{2}, S_{3}, U_{3}$ & $1100(800) \mathrm{GeV}$ & $1810(1540) \mathrm{GeV}$ & $35.9 \mathrm{fb}^{-1} \quad[75]$ \\
\hline$t \bar{t} \nu \bar{\nu}$ & $R_{2}, S_{3}, U_{1}, U_{3}$ & $1020(820) \mathrm{GeV}$ & $1780(1530) \mathrm{GeV}$ & $35.9 \mathrm{fb}^{-1} \quad[75]$ \\
\hline
\end{tabular}

Table 1. Summary of the current limits from LQ pair production searches at the LHC. In the first column we give the searched final states and in the second column the LQs for which this search is relevant. In the next two columns we present the current limits on the mass for scalar and vector LQs, respectively, for $\beta=1(\beta=0.5)$. In the last column we display the value of the LHC luminosity for each search along with the experimental references. Note that " $j$ " denotes any jet originating from a charm or a strange quark. Entries marked with "-" indicate that no recast or search in this channel has been performed up to this date.

$\beta=1(0.5)$. These limits assume the following: (i) pair production is dominated by QCD interactions, and (ii) for vector LQs $\left(V^{\mu}\right)$ the LQ-gluon interaction term, $\mathcal{L} \supset-\kappa g_{s} V^{\mu} G_{\mu \nu} V^{\nu}$, is taken with $\kappa=1$. The first assumption is in general true for LQ-fermion couplings of order $\sim 1$ or smaller [68]. In this regime, contributions to $q \bar{q} \rightarrow \mathrm{LQ}^{\dagger} \mathrm{LQ}$ with a $t$-channel lepton (where the amplitude is proportional to the squared LQ-fermion coupling) are subleading compared to QCD induced production. The assumption on the value of $\kappa$, instead, depends on the UV origin of the vector LQ [76]. If $V^{\mu}$ is a fundamental gauge boson of a new non-abelian gauge group then the gauge symmetry completely fixes the choice $\kappa=1$. The possibility of having $|\kappa|<1$ may arise in a UV theory where the vector LQ is a composite particle, therefore giving rise to LHC limits weaker than for the gauge vector LQ presented in table. 1.

\subsection{Limits from high- $p_{T}$ tails of $p p \rightarrow \ell \bar{\ell}$}

As shown in refs. [77, 78], a contribution arising from the $t$-channel exchange of LQs to $p p \rightarrow \ell \bar{\ell}(\ell=\mu, \tau)$ can be directly probed in the high- $p_{T}$ tails of Drell-Yan processes at the LHC. In particular, larger values of Yukawa couplings, that are often needed to accommodate the $B$-anomalies, could modify the tail of the differential cross section of $p p \rightarrow \ell \bar{\ell}$. In the following we use LHC data from $p p$-collisions at $13 \mathrm{TeV}$ to set limits on each LQ model. For this we have recast two recent searches by ATLAS at $36.1 \mathrm{fb}^{-1}$ for a $Z^{\prime}$ decaying to $\mu \bar{\mu}[79]$ and $\tau \bar{\tau}[80]$, respectively.

For the di-tau analysis we focused on the fully inclusive channel with hadronic taus $\left(\tau_{\text {had }}\right)$ in the final state,given that these perform considerably better at high $p_{T}$ than the 
leptonic tau decay channels. For each search we counted the number of observed and background events above different threshold values of the invariant mass distributions $m_{\mu \mu}$ for the di-muon search and the total transverse mass distribution $m_{\text {tot }}$ for the di-tau search (see ref. [80] for the definition of $m_{\text {tot }}$ ). An upper bound at 95\% C.L. on the number of allowed signal events above each mass threshold was extracted for each search by minimizing the Log-Likelihood ratio with nuisance parameters for the background uncertainties, as described in [81]. Besides the current luminosity limits, we also estimated projected limits at a higher LHC luminosity of $\mathcal{L}_{\text {int }}=300 \mathrm{fb}^{-1}$ by scaling the data and background events with the luminosity ratio and the background uncertainties with $\sqrt{\mathcal{L}_{\text {int }}}$ assuming that the data in the distribution tails are statistically dominated. This assumption holds well for the leading backgrounds such as SM Drell-Yan production or fake $\tau_{\text {had }}$ from QCD jet mis-tagging since these are estimated using experimental data from control regions that improve with more statistics. Additionally, systematics in the tails of the di-muon and di-tau searches are well under control and only dominate the lower bins where the search is insensitive to the massive LQs.

For our simulations we created the Universal FeynRules Output (UFO) files using FeynRules [82] for each LQ mediator $\left(S_{1}, S_{3}, R_{2}, U_{1}\right.$ and $\left.U_{3}\right)$ coupling exclusively to second and third generation of quarks and leptons. For $R_{2}$ and $U_{1}$ we have only considered, for simplicity, non-zero left-handed Yukawa matrices in eq. (3.6) and eq. (3.17). After exporting the UFOs to MadGraph5 [83] we generated for each LQ mediator a statistically significant set of $t$-channel Drell-Yan event samples $q \bar{q} \rightarrow \mu \bar{\mu}, \tau \bar{\tau}$ for initial sea quarks, $q \in\{s, c, b\}$, and for vector (scalar) LQs at different masses in the $1-6 \mathrm{TeV}(0.6-3 \mathrm{TeV})$ range. Each sample was subsequently showered and hadronized using Pythia8 [84]. Final state hadronic taus and isolated muons where reconstructed and smeared using Delphes3 [85] with the parameters set according to each experimental scenario. In order to illustrate the current reach of the LHC for each LQ state we show in figure 4 results from the $p p \rightarrow \mu \bar{\mu}$ and $p p \rightarrow \tau \bar{\tau}$ searches by ATLAS for scalar (vector) LQs in the $y_{L}^{q l}\left(x_{L}^{q l}\right)$ coupling versus mass plane. ${ }^{9}$ Each line corresponds to the $95 \%$ upper limit for the process $q \bar{q} \rightarrow \ell \bar{\ell}$ turning on each flavor coupling one at a time, with $q \in\{s, c, b\}$ and $\ell \in\{\mu, \tau\}$. Similar bounds of the same order can be extracted for the coupling products $y_{L}^{q l} y_{L}^{q^{\prime} l}$ and $x_{L}^{q l} x_{L}^{q^{\prime} l}$ with $q \neq q^{\prime}$ from the quark flavor violating process $q q^{\prime} \rightarrow \ell \bar{\ell}$.

Besides producing deviations in the di-lepton tails, LQ mediators that couple simultaneously to differently charged leptons may also produce measurable effects in LFV observables at the LHC. In particular, searching for the process $p p \rightarrow \ell \ell^{\prime}$ with a LQ exchanged in the $t$-channel may provide an additional handle for setting constraints on the flavor structure of these LQ models. Existing searches for a massive $Z^{\prime}$ with LFV couplings have been presented by the LHC collaborations in the $Z^{\prime} \rightarrow e \mu, e \tau, \mu \tau$ channels. In order to determine the sensitivity of the LHC to the $t$-channel process $p p \rightarrow \mu \tau$ we recast the LFV $Z^{\prime}$ search by ATLAS $[86,87]$ at $3.2 \mathrm{fb}^{-1}$. We find, however, that the bounds on the LQs extracted from the high- $p_{T} m_{\mu \tau}$ tails are always weaker than the combined bounds from the flavor conserving di-muon and di-tau tails described above.

\footnotetext{
${ }^{9}$ We did not present plots for $S_{1}$ since these bounds are identical to the bounds for $S_{3}^{1 / 3}$.
} 

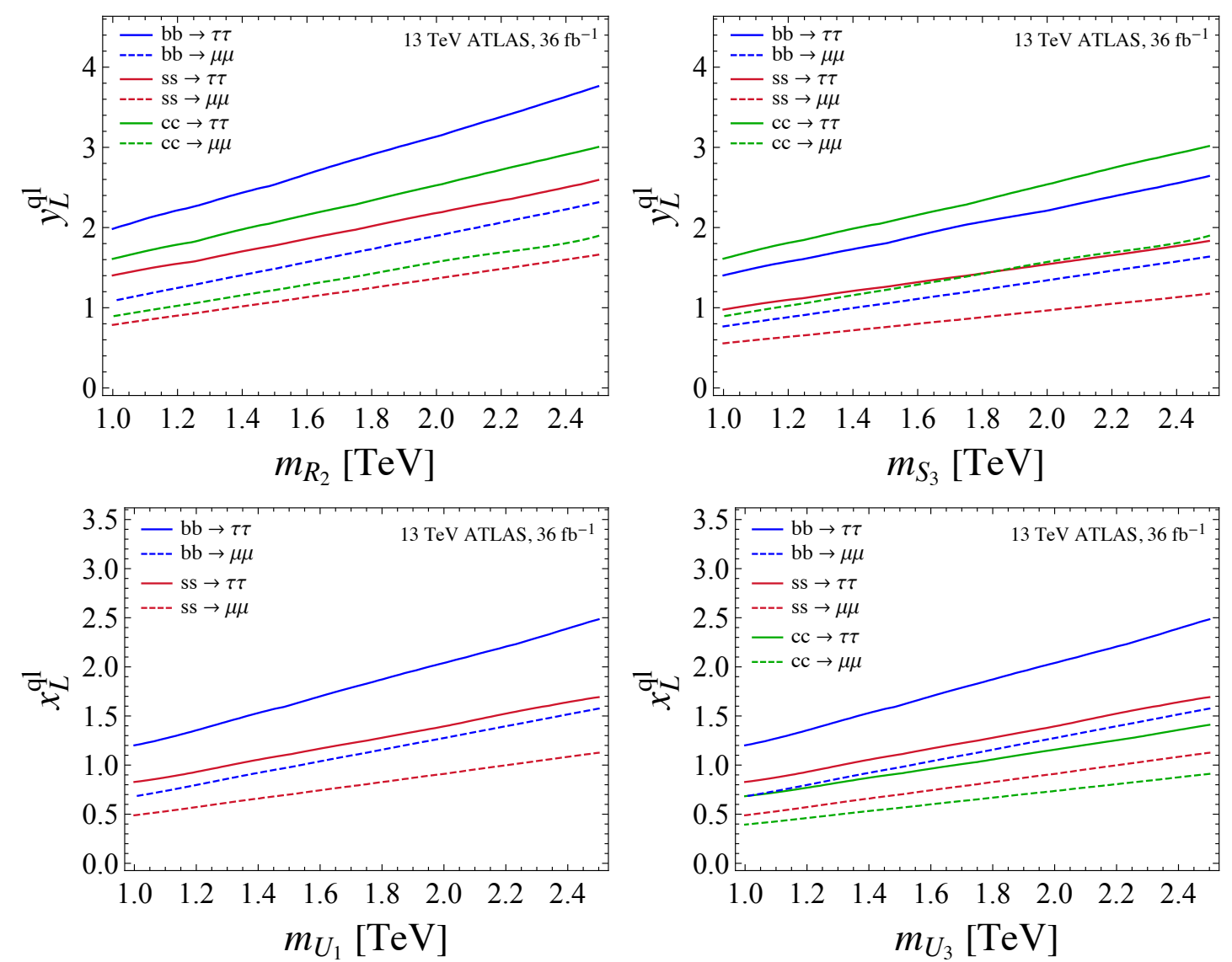

Figure 4. The top panel (lower panel) shows current limits in the coupling vs mass plane for several scalar LQ (vector LQ) models from LHC searches in $p p \rightarrow \ell \bar{\ell}$ high- $p_{T}$ tails at $13 \mathrm{TeV}$ with $36 \mathrm{fb}^{-1}$ of data. The solid and dashed lines represent limits from di-tau and di-muon searches, respectively, for different initial quarks while turning one scalar (vector) LQ coupling $y_{L}^{q l}\left(x_{L}^{q l}\right)$ at a time.

\section{$5 \quad$ Which leptoquark model?}

In this section we subject the models listed in section 3 to the constraints stemming both from direct and indirect NP searches. We will then select LQ scenarios which can accommodate $R_{K^{(*)}}^{\exp }$ and/or $R_{D^{(*)}}^{\exp }$ based on the expressions derived above.

- $S_{3}=(\overline{\mathbf{3}}, \mathbf{3})_{1 / 3}$ :

As already discussed in section 3.1.1, the $S_{3}$ model can accommodate $R_{K^{(*)}}^{\exp }$ since it predicts the NP contribution to $C_{9,10}$ satisfying $C_{9}=-C_{10}$. On the other hand, this scenario cannot accommodate the anomalies in $R_{D^{(*)}}^{\exp }>R_{D^{(*)}}^{\mathrm{SM}}$. This can be easily understood from eq. (3.4), where we see that the sign of the leading term for $g_{V_{L}}$ is negative, thus further lowering the value of $R_{D^{(*)}}^{\mathrm{SM}}$, see also eq. (2.5). The subleading terms in this equation could in principle provide a positive contribution to $R_{D^{(*)}}$ but such a situation would be in conflict with tight constraints coming from other flavor physics limits, such as $B \rightarrow K \nu \nu$ and $\Delta m_{B_{s}}$. 
- $R_{2}=(3,2)_{7 / 6}$ :

At tree level the $R_{2}$ model contributes to $C_{9,10}$ in such a way that they satisfy $C_{9}=C_{10}$, which disagrees with $R_{K^{(*)}}<R_{K^{(*)}}^{\mathrm{SM}}$, cf. section 3.1.2. This situation can be avoided by choosing a flavor structure such that the tree-level contribution to $b \rightarrow s \mu \mu$ is absent. As a result, $C_{9,10}$ are loop induced and the NP Wilson coefficients satisfy $C_{9}=-C_{10}$, as needed [35]. The minimal flavor ansatz in eq. (3.6) to realize such a scenario is given by

$$
y_{L}=\left(\begin{array}{ccc}
0 & 0 & 0 \\
0 & y_{L}^{c \mu} & 0 \\
0 & y_{L}^{t \mu} & 0
\end{array}\right), \quad y_{R}=0
$$

where $y_{L}^{c \mu}$ and $y_{L}^{t \mu}$ are non-zero couplings. For the time being, we neglected the coupling to $\tau$ because it plays no role in the discussion of $R_{K^{(*)}}$. The main challenge in this scenario is to comply with LHC limits, which are particularly constraining in the region the anomalies can be accounted for. To see the extent to which the model can accommodate measured $R_{K^{(*)}}$ we use the allowed values of Wilson coefficients specified in eq. (2.3), as well as the constraints arising from the limits on $\mathcal{B}\left(B \rightarrow K^{(*)} \nu \bar{\nu}\right)$ and from the $Z$-pole observables [52]. The results of our fit are then combined with limits derived in section 4 , and shown in figure (5), for two benchmark masses, $m_{R_{2}}=1.2 \mathrm{TeV}$ and $1.5 \mathrm{TeV}$, cf. table 1 . From this plot we see that a large $y_{L}^{c \mu}$ needed at low-energies is in tension with LHC limits, allowing then an explanation of $R_{K^{(*)}}$ only at the $2 \sigma$ level. Therefore, if the central values of $R_{K}^{\exp }$ and $R_{K^{*}}^{\exp }$ remain unchanged with more data, this model will be excluded as an explanation of the $b \rightarrow s$ anomalies. A similar conclusion has been independently found in ref. [73].

The above flavor pattern is clearly not satisfactory to explain the observed $R_{D^{(*)}}>$ $R_{D^{(*)}}^{\mathrm{SM}}$. To accommodate those one could proceed as in refs. $[35,54,55,88]$ and let $y_{L}^{c \tau}\left(y_{R}^{b \tau}\right)^{*}$ to be of order $\mathcal{O}(1)$, which is compatible both with low-energy observables and with upper limits on $p p \rightarrow \tau \tau$ derived at LHC. This is illustrated in figure 4. From the point of view of the effective Lagrangian (2.4) this LQ scenario generates the combination $g_{S_{L}}=4 g_{T}$ at the matching scale $\mu \simeq 1 \mathrm{TeV}$, which can turn $R_{D^{(*)}}$ compatible with $R_{D^{(*)}}^{\exp }$ if $g_{S_{L}}$ is mostly imaginary, cf. figure 2 . We should reiterate that a simultaneous explanation of both $R_{K^{(*)}}$ and $R_{D^{(*)}}$ cannot be obtained in this scenario even at the $2 \sigma$ level. This is so because the couplings required to accommodate each of the observed anomalies would induce too large a value for $\mathcal{B}(\tau \rightarrow \mu \gamma)$, due to the chiral enhancement by the top quark [52].

- $\widetilde{R}_{2}=(\mathbf{3}, \mathbf{2})_{1 / 6}$ :

In a model with the LQ state $\widetilde{R}_{2}$, the non-zero NP Wilson coefficients in the $b \rightarrow s \mu \mu$ decay are those corresponding to the chirality flipped operators and they satisfy $C_{9}^{\prime}=-C_{10}^{\prime}$. While this is enough to explain $R_{K}<R_{K}^{\mathrm{SM}}$, it predicts $R_{K^{*}}>R_{K^{*}}^{\mathrm{SM}}$ which is in disagreement with experimental data [56]. In ref. [58] this model has also been considered to account for the anomalies in charged currents. To this purpose, 

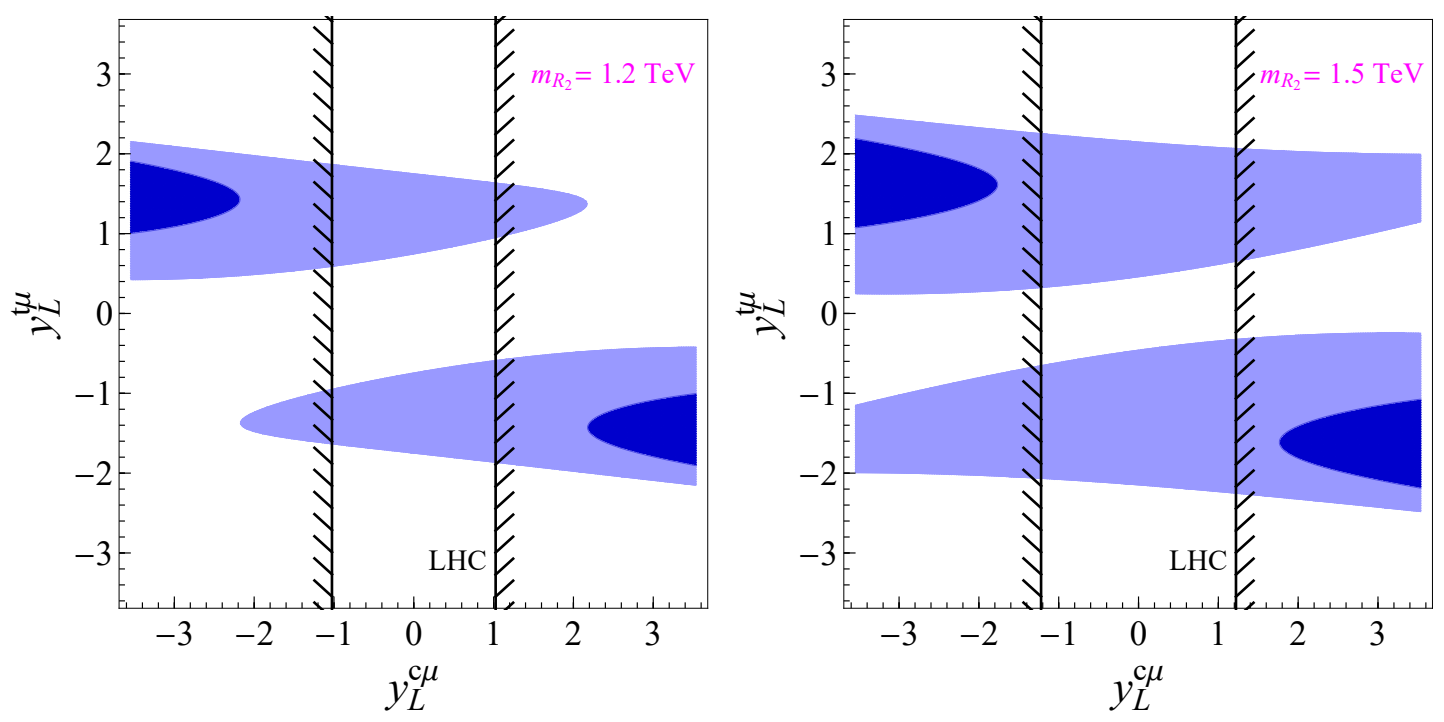

Figure 5. Regions in plane $y_{c \mu}$ vs. $y_{t \mu}$ with $\Delta \chi^{2}=2.3(6.8)$ are plotted in dark (light) blue for $m_{R_{2}}=1.2 \mathrm{TeV}$ (left panel) and $1.5 \mathrm{TeV}$ (right panel). We consider the $b \rightarrow$ sll constraint [eq. (2.3)] and the ones from the $Z$-pole observables computed in [52]. The black line denotes the LHC limits derived from $p p \rightarrow \mu \mu$ data with $36 \mathrm{fb}^{-1}$, which excludes an explanation of $R_{K^{(*)}}$ to $1 \sigma$ for both masses. See text for details.

it is necessary to postulate the existence of light right-handed neutrinos, as already mentioned in section 3.1.3. The main difficulty of this scenario is to face the LHC limits on (i) recent limits on pair-produced LQs given in table 1, and (ii) $\tau \tau$ dilepton tails [77]. The combination of these latter constraints is particularly efficient in this scenario, so that $\widetilde{R}_{2}$ model can only account for a very small enhancement in $R_{D^{(*)}}$.

- $S_{1}=(\overline{\mathbf{3}}, \mathbf{1})_{1 / 3}$ :

The $S_{1}$ model is a viable candidate to explain $R_{D^{(*)}}>R_{D^{(*)}}^{\mathrm{SM}}$ since it generates the effective couplings $g_{V_{L}}$ and $g_{S_{L}}=-4 g_{T}$, both being equally viable when it comes down to accommodating $R_{D^{(*)}}^{\exp }>R_{D^{(*)}}^{\mathrm{SM}}$, see figure 2 . On the other hand, this scenario does not provide a tree-level contribution to $b \rightarrow$ sll but $C_{9,10}$ can be induced by the box-diagrams satisfying $C_{9}=-C_{10}$, as desired [59]. It appears, however, that this is not a viable scenario if one works with $m_{S_{1}} \approx 1 \mathrm{TeV}$ and if one wants to stay compatible with $R_{D^{(*)}}^{\mu / e}=\mathcal{B}\left(B \rightarrow D^{(*)} \mu \bar{\nu}\right) / \mathcal{B}\left(B \rightarrow D^{(*)} e \bar{\nu}\right)$, as discussed in ref. [20]. A step beyond was made in ref. [89] in which the model was shown to be viable if (i) a larger LQ mass is considered, and (ii) a different coupling structure, with large right-handed LQ couplings to the $\tau$-lepton, is allowed.

To evaluate the extent to which this particle can improve the description of the $b \rightarrow s$ anomalies, we consider the minimal flavor ansatz,

$$
y_{L}=\left(\begin{array}{ccc}
0 & 0 & 0 \\
0 & y_{L}^{s \mu} & 0 \\
0 & y_{L}^{b \mu} & 0
\end{array}\right), \quad y_{R}=0
$$


where we keep only the couplings that contribute to $C_{9}=-C_{10} \cdot{ }^{10}$ We then perform a fit to the low-energy observables modified by these couplings, namely, (i) the values of Wilson coefficients given in eq. (2.3), (ii) the experimental limits on $\mathcal{B}(B \rightarrow K \nu \bar{\nu})$, (iii) the experimentally established $\Delta m_{B_{s}}$, (iv) $Z$-boson couplings to leptons measured at LEP, (v) leptonic decays $D_{(s)} \rightarrow \mu \bar{\nu}$ and $K \rightarrow \mu \bar{\nu}$, and (vi) the rare charm decay $D^{0} \rightarrow \mu \mu$. All the expression needed for that analysis, together with the experimental values/bounds can be found in ref. [20]. We find $\chi_{\min }^{2} /$ d.o.f. $=11.2 / 3$ for the following choice of parameters:

$$
y_{L}^{b \mu} \approx \sqrt{4 \pi}, \quad y_{L}^{s \mu} \approx-0.15, \quad m_{S_{1}} \approx 5.2 \mathrm{TeV},
$$

where we have imposed the perturbativity limit $\left|y_{L}^{i j}\right| \leq \sqrt{4 \pi}$. The value $\chi_{\min }^{2}=11.2$ is smaller than the $\mathrm{SM}$ value, $\chi_{\mathrm{SM}}^{2}=21.2$, showing that the discrepancy can be decreased but not fully accommodated. In figure 6 we plot the regions with $\Delta \chi^{2}<2.3$ and 6.8 in the planes $m_{S_{1}}$ vs. $y_{L}^{s \mu}$ and $m_{S_{1}}$ vs. $y_{L}^{b \mu}$. From this plot we see that one needs large LQ masses and large $\left|y_{L}^{b \mu}\right|$ to satisfactorily accommodate the current data. Such large couplings are close to the nonperturbative regime $\left|y_{L}^{b \mu}\right| \gtrsim \sqrt{4 \pi}$, indicating that one needs a systematic study of one-loop corrections before assessing the viability of this scenario. In figure 6 we also show the bounds coming from the direct searches at LHC which are weak in the region of parameters described above. By assuming $\left|y_{L}^{b \mu}\right| \gg\left|y_{L}^{s \mu}\right|$, the bound we deduce from the pair production searches in the $t \bar{t} \mu \bar{\mu}$ channel is $m_{S_{1}}>1.4 \mathrm{TeV}$ [74], much lower than the one we get from the flavor fit, given in eq. (5.3). We should note that limits from the study of dilepton tails, as described in section 4.2 , are too weak, as expected, because the flavor fit favors couplings mostly to the third generation of quarks, for which the PDFs are very suppressed.

So far we were concerned with the $b \rightarrow s \ell l$ anomalies, $R_{K^{(*)}}$. If instead we focus on $R_{D^{(*)}}$ then the flavor ansatz given in eq. (5.2) must be extended to allow for $y_{R}^{c \tau} \neq 0$ :

$$
y_{L}=\left(\begin{array}{ccc}
0 & 0 & 0 \\
0 & y_{L}^{s \mu} & y_{L}^{s \tau} \\
0 & y_{L}^{b \mu} & y_{L}^{b \tau}
\end{array}\right), \quad y_{R}=\left(\begin{array}{ccc}
0 & 0 & 0 \\
0 & 0 & y_{R}^{c \tau} \\
0 & 0 & y_{R}^{t \tau}
\end{array}\right)
$$

The couplings to $\tau$ bring in new constraints, such as those coming from $\mathcal{B}\left(D_{s} \rightarrow \tau \bar{\nu}\right)$, $\mathcal{B}(B \rightarrow \tau \nu)$ and $\mathcal{B}(\tau \rightarrow \mu \gamma)$, as described in ref. [20]. By performing a $\chi^{2}$ analysis similar to the one above we conclude that the tension in $R_{K^{(*)}}$ and $R_{D^{(*)}}$ can be moderately reduced if one is to remain in the range of $m_{S_{1}} \lesssim 2 \mathrm{TeV}$. To accommodate them one would need large couplings, $\left|y_{L}^{b \mu}\right| \approx \sqrt{4 \pi}$ and $\left|y_{R}^{c \tau}\right| \approx \sqrt{4 \pi}$, and $m_{S_{1}} \gtrsim 4 \mathrm{TeV}$, which is different from the original proposal in ref. [59]. These conclusions agree with ref. [89].

\footnotetext{
${ }^{10}$ Note that the couplings $y_{R}^{i j}$ to right-handed leptons will generate the combination of Wilson coefficients $C_{9}+C_{10}$, which is disfavored by the current data.
} 

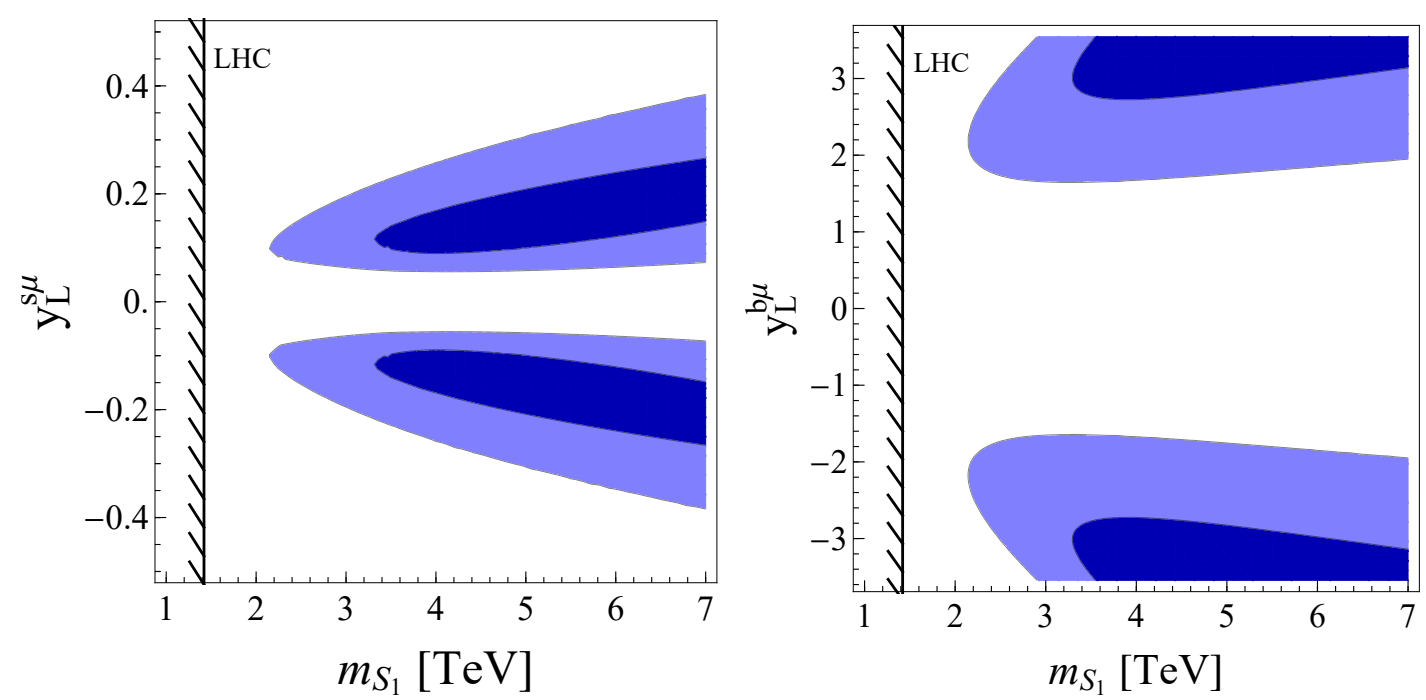

Figure 6. $m_{S_{1}}$ is plotted against $y_{L}^{s \mu}$ (left panel) and $y_{L}^{b \mu}$ (right panel). Light (dark) blue regions correspond to $\Delta \chi^{2}<2.3(6.8)$. The black line in the right panel denotes the LHC exclusion limit on pair produced LQs decaying into $t \mu$ [74]. See text for details.

- $U_{1}=(\mathbf{3}, \mathbf{1})_{2 / 3}$ :

As discussed in ref. [60] the minimal $U_{1}$ model is one of the best candidates to simultaneously explain the anomalies in $R_{K^{(*)}}$ and $R_{D^{(*)}}$. The interesting features of this scenario are: (i) contributions to $R_{K^{(*)}}$ come from the Wilson coefficients $C_{9,10}$ satisfying $C_{9}=-C_{10}$, (ii) a positive sign of $g_{V_{L}}$ allows to accommodate $R_{D^{(*)}}>R_{D^{(*)}}^{\mathrm{SM}}$, cf. eq. (3.19), and (iii) the absence of tree-level contributions to $\mathcal{B}\left(B \rightarrow K^{(*)} \nu \bar{\nu}\right)$, which is often a major obstacle to the models built to accommodate the $B$-physics anomalies. We will discuss this scenario in more detail in section 6 .

- $U_{3}=(\mathbf{3}, \mathbf{3})_{2 / 3}$ :

Finally, the model $U_{3}$ is also a viable candidate to explain $R_{K^{(*)}}$, since it predicts $C_{9}=-C_{10}$, but it cannot provide an explanation of $R_{D^{(*)}}>R_{D^{(*)}}^{\mathrm{SM}}$. This can be understood on the basis of eq. (3.22) from which we learn that the leading contribution to $g_{V_{L}}$ comes with the wrong (negative) sign. The subleading couplings to the strange quark are constrained by the tight experimental limits on $\mathcal{B}\left(B \rightarrow K^{(*)} \nu \bar{\nu}\right)$, which is why the net effect on $R_{D^{(*)}}$ is very small.

Our findings are summarized in table 2, from which we learn that $U_{1}$ is the only single LQ model that can simultaneously accommodate $R_{K^{(*)}}$ and $R_{D^{(*)}}$, in agreement with findings of ref. [60]. A slightly non-minimalistic possibility is to build a model with two different scalar leptoquarks, as explored for $S_{1}$ and $S_{3}$ in refs. [60, 90, 91], and for $R_{2}$ and $S_{3}$ in ref. [88]. Note that our conclusions can also serve as a guideline for future studies if one of the anomalies disappears. 


\begin{tabular}{|c|c|c|c|}
\hline Model & $R_{K^{(*)}}$ & $R_{D^{(*)}}$ & $R_{K^{(*)}} \& R_{D^{(*)}}$ \\
\hline$S_{1}$ & $x^{*}$ & $\checkmark$ & $x^{*}$ \\
$R_{2}$ & $x^{*}$ & $\checkmark$ & $x$ \\
$\widetilde{R_{2}}$ & $x$ & $x$ & $x$ \\
$S_{3}$ & $\checkmark$ & $x$ & $x$ \\
\hline$U_{1}$ & $\checkmark$ & $\checkmark$ & $\checkmark$ \\
$U_{3}$ & $\checkmark$ & $x$ & $x$ \\
\hline
\end{tabular}

Table 2. Summary of the LQ models which can accommodate $R_{K^{(*)}}$ (first column), $R_{D^{(*)}}$ (second column), and both $R_{K^{(*)}}$ and $R_{D^{(*)}}$ (third column) without inducing other phenomenological problems. The symbol $\boldsymbol{X}^{*}$ means that the discrepancy can be alleviated, but not fully accommodated. See text for details.

\section{Revisiting $U_{1}=(3,1)_{2 / 3}$}

In this section we discuss in more detail the phenomenological status of the scenario $U_{1}$. We will use the low-energy physics observables which receive the tree-level contributions from the $U_{1}$ exchange to constrain the model parameters. We will also compare these results with the ones deduced from the experimental bounds based on direct searches at the LHC. Furthermore, we will make a brief comment concerning the loop effects.

\subsection{Low-energy constraints}

To satisfy both $R_{K^{(*)}}<R_{K^{(*)}}^{\mathrm{SM}}$ and $R_{D^{(*)}}>R_{D^{(*)}}^{\mathrm{SM}}$ we will assume the following structure for the Yukawa matrices:

$$
x_{L}=\left(\begin{array}{ccc}
0 & 0 & 0 \\
0 & x_{L}^{s \mu} & x_{L}^{s \tau} \\
0 & x_{L}^{b \mu} & x_{L}^{b \tau}
\end{array}\right), \quad \quad x_{R}=0
$$

where the couplings to the first generation are set to zero in order to avoid the conflicts with experimental limits on $\mu-e$ conversion on nuclei, the atomic parity violation and on $\mathcal{B}(K \rightarrow \pi \nu \bar{\nu})$. To determine the region allowed by $R_{K^{(*)}}^{\exp }$, we compare the result of the global fit to $b \rightarrow s \mu \mu$ observables, given in eq. (2.3), with the expression eq. (3.18), and find

$$
-\frac{x_{L}^{s \mu}\left(x_{L}^{b \mu}\right)^{*}}{m_{U_{1}}^{2}} \in[0.83,1.41] \times 10^{-3} \mathrm{TeV}^{-2} .
$$

A tree-level contribution of $U_{1}$ to $R_{D^{(*)}}$ gives rise to the effective coefficient $g_{V_{L}}$, cf. eq. (3.19), which compared to eq. (2.5) results in

$$
\frac{\left(V x_{L}\right)_{c \tau}\left(x_{L}^{b \tau}\right)^{*}}{m_{U_{1}}^{2}}=\frac{\left(V_{c s} x_{L}^{s \tau}+V_{c b} x_{L}^{b \tau}\right)\left(x_{L}^{b \tau}\right)^{*}}{m_{U_{1}}^{2}} \in[0.12,0.18] \mathrm{TeV}^{-2} .
$$




\begin{tabular}{|c|c|c|c|}
\hline Observable & Eqs. $-U_{1}$ & Exp. value & Ref. \\
\hline$b \rightarrow s \mu \mu$ & $(3.18)$ & $(2.3)$ & {$[25-28]$} \\
$b \rightarrow c \tau \nu$ & $(3.19)$ & $(2.5)$ & {$[8]$} \\
$\mathcal{B}(\tau \rightarrow \mu \phi)$ & $(6.4)$ & $<8.4 \times 10^{-8}$ & {$[92]$} \\
$\mathcal{B}(B \rightarrow \tau \nu)$ & $(3.19),(2.6)$ & $1.06(19) \times 10^{-4}$ & {$[8]$} \\
$\mathcal{B}\left(D_{s} \rightarrow \mu \nu\right)$ & $(3.19),(2.6)$ & $5.50(23) \times 10^{-3}$ & {$[92]$} \\
$\mathcal{B}\left(D_{s} \rightarrow \tau \nu\right)$ & $(3.19),(2.6)$ & $5.48(23) \times 10^{-2}$ & {$[92]$} \\
$r_{K}^{e / \mu}$ & $(3.19),(2.6)$ & $2.488(10) \times 10^{-5}$ & {$[92,97]$} \\
$r_{K}^{\tau / \mu}$ & $(3.19),(2.6)$ & $4.670(67) \times 10^{2}$ & {$[48]$} \\
$R_{D}^{\mu / e}$ & $(3.19)$ & $0.995(22)(39)$ & {$[98]$} \\
$\mathcal{B}(B \rightarrow K \mu \tau)$ & $(3.18)$ & $<4.8 \times 10^{-5}$ & {$[99]$} \\
\hline
\end{tabular}

Table 3. Tree-level observables considered in our phenomenological analysis and their corresponding experimental values (or limits), as well as their theoretical expressions for the $U_{1}$ scenario.

Other relevant constraints to this scenario are listed in table 3, including the decays $K \rightarrow$ $\mu \bar{\nu}, D_{(s)} \rightarrow \tau \bar{\nu}$ and $B \rightarrow \tau \bar{\nu}$, as well as the ratio $R_{D}^{\mu / e}=\mathcal{B}(B \rightarrow D \mu \bar{\nu}) / \mathcal{B}(B \rightarrow D e \bar{\nu})$. Another important constraint stems from $\mathcal{B}(\tau \rightarrow \mu \phi)^{\exp }<8.4 \times 10^{-8}[92,93]$, which was often neglected in previous studies of this particular LQ model, and which gives (to $2 \sigma$ ),

$$
\frac{\left|x_{L}^{s \mu}\right|\left|x_{L}^{s \tau}\right|}{m_{U_{1}}^{2}}<0.018 \mathrm{TeV}^{-2}
$$

We reiterate that an important feature of this scenario is the absence of tree-level contributions to $B \rightarrow K^{(*)} \nu \bar{\nu}$. One-loop contributions to this transition as well as to many other observables, such as $\tau \rightarrow \mu \gamma, B_{s}-\bar{B}_{s}$ mixing and LFU tests in $\tau$ decays, can however be important. In refs. [94-96] it was shown that the leading-log renormalization group effects induced by the effective operators related to $U_{1}$ could be in conflict with constraints from leptonic $Z$ and $\tau$ decays in the scenarios in which the dominant coupling is the one to the third generation fermions. To get around that difficulty one can allow for a more general flavor structure [60], similar to the one we consider here. In this work, we will not consider the constraints induced by the loop effects since they could be sensitive to the details of the unknown UV completion of the $U_{1}$ model, which is model dependent. We will simply assume that this problem is taken care of by some mechanism which prevents the appearances of divergences to higher order in perturbation theory. Moreover, as we shall see below, the synergy between the tree-level constraints from flavor physics and the tree-level bounds coming from direct searches at the LHC is already sufficient to significantly limit the parameter space of this scenario. Of course, this fact does not reduce importance of electroweak corrections which must be systematically included in the models in which the UV completion is specified. 


\subsection{Results and predictions}

The results of our analysis will be presented in two parts. In the first we set $m_{U_{1}}=1.5 \mathrm{TeV}$, which is the lowest $U_{1}$ mass not yet excluded by vector LQ pair production searches at the LHC [75]. The resulting parameter space will then be used to show our predictions for two LFV processes, $B \rightarrow K \mu \tau$ and $\tau \rightarrow \mu \phi$. In the second part, we repeat the same exercise but this time treating $m_{U_{1}}$ as a free parameter.

Scan of parameters with $\boldsymbol{m}_{U_{1}}=1.5 \mathrm{TeV}$. For our analysis with fixed $m_{U_{1}}=1.5 \mathrm{TeV}$, we first find a best fit point by minimizing a $\chi^{2}$-function built from the flavor observables listed in table 3 . We find $\chi_{\min }^{2}=6.61$ for

$$
x_{L}^{s \mu} \approx-10^{-2}, \quad x_{L}^{b \mu} \approx 0.25, \quad x_{L}^{s \tau} \approx 4.4 \times 10^{-3}, \quad x_{L}^{b \tau} \approx 2.81 .
$$

We then perform a random scan over the values of the four left-handed couplings shown in eq. (6.1), and enforce perturbativity, $\left|x_{L}^{i j}\right| \leq \sqrt{4 \pi}$. We select only the points which satisfy $\Delta \chi^{2}$ (par) $\equiv \chi^{2}$ (par) $-\chi_{\min }^{2} \leq 6.18$, i.e. within $2 \sigma$ from the best fit point. The selected points are compared with the limits deduced from the direct LHC searches in $\ell \ell$ $(\ell=\mu, \tau)$ final states, as detailed in section 4.2. In the plots presented in this section, the points excluded by direct searches based on current LHC data $\left(36 \mathrm{fb}^{-1}\right)$ are shown in grey. Furthermore, the red points are those that are excluded from our projections to $300 \mathrm{fb}^{-1}$. The blue points are those that would survive.

We first show, in figure 7, the correlation between the two LQ couplings entering eq. (3.19) for $m_{U_{1}}=1.5 \mathrm{TeV}$. One observes that the experimental value of $R_{D^{(\star)}}$ forces $x_{L}^{b \tau}$ to be different from zero, thus bounding its absolute value from below. Even though the measurements of low-energy observables allow for $x_{L}^{s \tau}=0$, we see that current LHC data exclude this possibility, imposing the lower bound $\left|x_{L}^{s \tau}\right| \gtrsim 0.03$. Moreover, our projected bound for $300 \mathrm{fb}^{-1}$ will push this limit even further away from 0 , implying $\left|x_{L}^{s \tau}\right| \gtrsim 0.1$. Even though we opted to not consider the model dependent radiative bounds, which are derived within the leading logarithm approximation [94-96], it is interesting to note that comparable lower bounds on $\left|x_{L}^{s \tau}\right|$ can be obtained by these means [60].

Next, we show in figure 8 the correlations between $x_{L}^{b \mu}$ and $x_{L}^{s \tau}$ (left panel) and between $x_{L}^{b \tau}$ and $x_{L}^{s \mu}$ (right panel). The color code remains the same as before and the red (blue) dashed lines correspond to the LHC limits obtained at $36 \mathrm{fb}^{-1}\left(300 \mathrm{fb}^{-1}\right)$, but assuming for simplicity that the couplings which are not present in a given plot are set to zero. ${ }^{11} \mathrm{It}$ is clear from eq. (3.18) that in order to explain the measured deviation with respect to the $\mathrm{SM}$ in the $b \rightarrow s \mu \mu$ transitions, both $x_{L}^{s \mu}$ and $x_{L}^{b \mu}$ need to be different from zero. Moreover, as discussed in the case of figure 7, current and future LHC limits provide a lower bound on $\left|x_{L}^{s \tau}\right|$, while $R_{D^{(*)}}^{\exp }$ sets a lower limit on $\left|x_{L}^{b \tau}\right|$. These considerations have an important impact on the LFV decays, as we discuss below.

We finally show in figure 9 our prediction for the correlation of two LFV observables, $\mathcal{B}(\tau \rightarrow \mu \phi)$ and $\mathcal{B}(B \rightarrow K \mu \tau)$, with the hatched black lines denoting the current experimen-

\footnotetext{
${ }^{11}$ Setting other couplings to zero to get the dashed regions in these plots is the reason why some of the red points remain within the dashed blue rectangles (because for these points the other couplings which are not in the plot are not set to zero).
} 


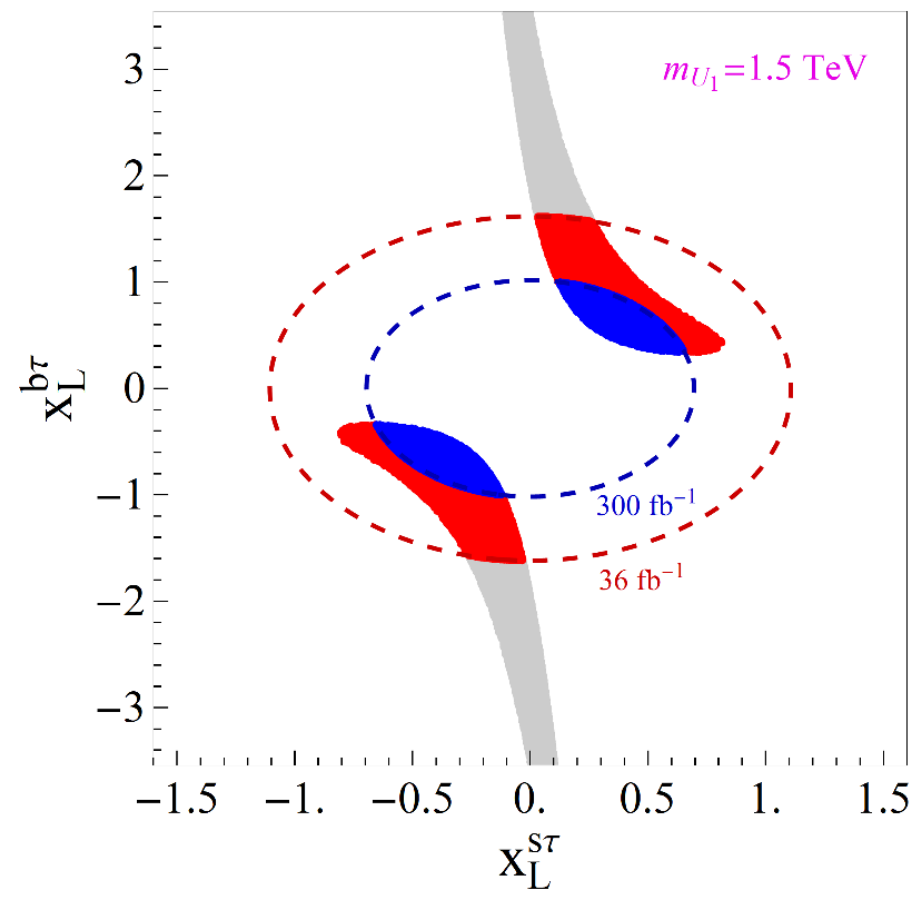

Figure 7. The correlation between the couplings $x_{L}^{s \tau}$ and $x_{L}^{b \tau}$ allowed by flavor constraints is plotted for $m_{U_{1}}=1.5 \mathrm{TeV}$. Gray points are excluded by current LHC data $\left(36 \mathrm{fb}^{-1}\right)$ on $p p \rightarrow \ell \ell(\ell=\mu, \tau)$. The future LHC sensitivity is depicted by the red points, which were obtained by extrapolating current data to $300 \mathrm{fb}^{-1}$, as discussed in section 4.2. Blue points are allowed by all constraints, including the extrapolated LHC results to $300 \mathrm{fb}^{-1}$.
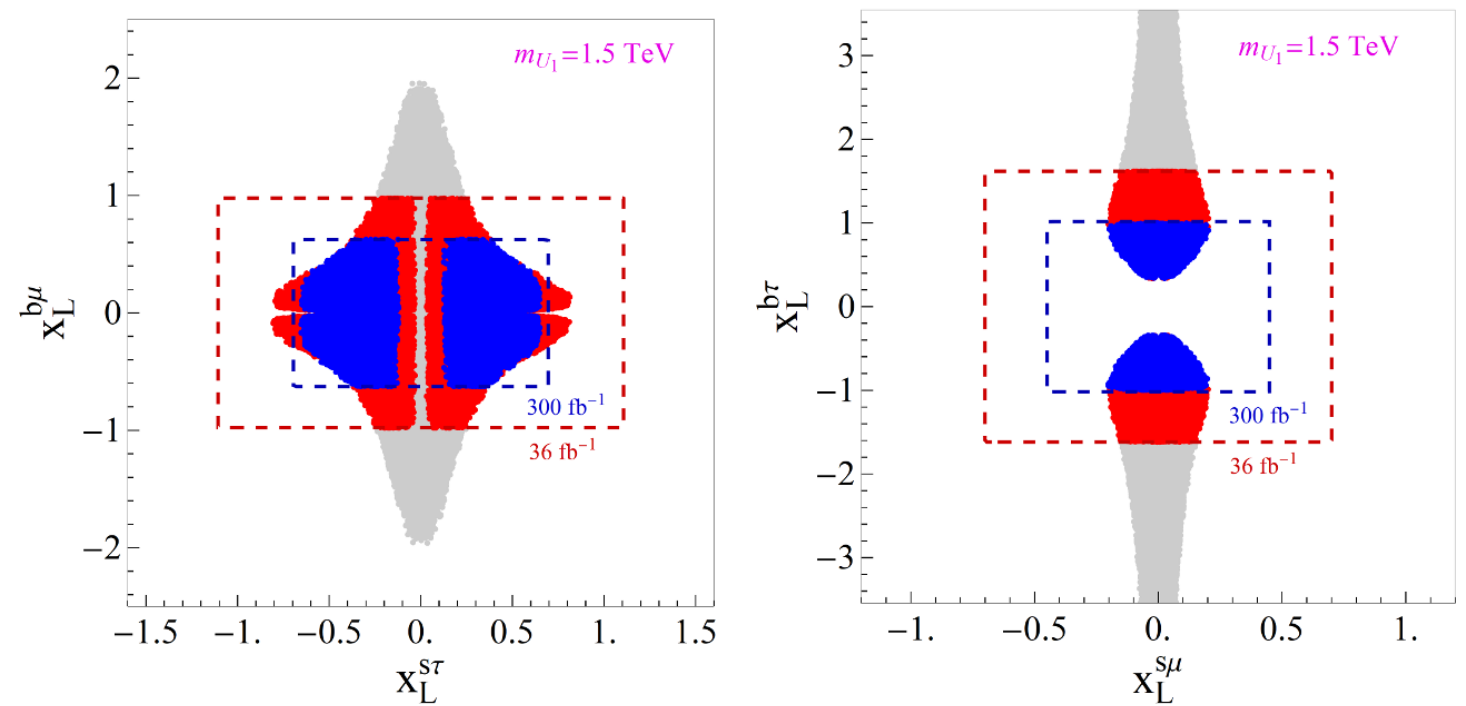

Figure 8. Coupling $x_{L}^{s \tau}$ is plotted against $x_{L}^{b \mu}$ (left panel), and $x_{L}^{b \tau}$ is plotted against $x_{L}^{s \mu}$ (right panel), by assuming $m_{U_{1}}=1.5 \mathrm{TeV}$. Color code is the same as in figure 7 . 


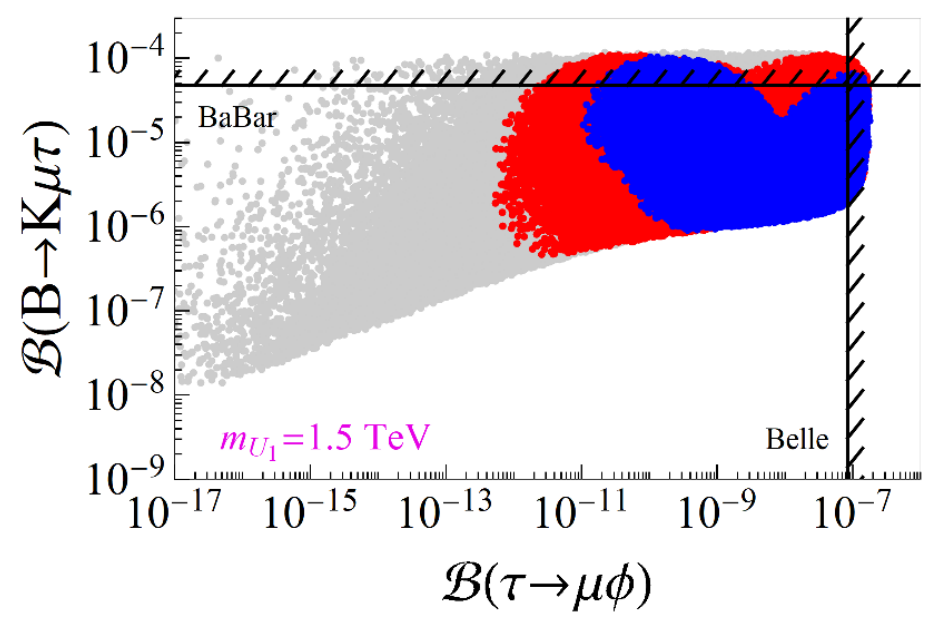

Figure 9. $\mathcal{B}(B \rightarrow K \mu \tau)$ is plotted against $\mathcal{B}(\tau \rightarrow \mu \phi)$ for the $U_{1}$ model. Color code is the same as in figure 7. Current bounds on these two decays, as respectively established by BaBar [99] and by Belle [93], are also shown.

tal bounds on these processes. Again, $m_{U_{1}}$ is set to $1.5 \mathrm{TeV}$. As mentioned in the previous paragraph, the fact that the LHC sets a lower bound on the absolute value of $\left|x_{L}^{s \tau}\right|$ has a dramatic impact on the amount of LFV predicted by the $U_{1}$ model: as can be seen in figure 9. Interestingly, we see that the current LHC bounds lead to $\mathcal{B}(B \rightarrow K \mu \tau) \gtrsim 2 \times 10^{-7}$, which remains rather stable lower bound for the LFV mode. With $300 \mathrm{fb}^{-1}$ we get that this bound is improved to $\mathcal{B}(B \rightarrow K \mu \tau) \gtrsim 5 \times 10^{-7}$. In other words, we get an absolute lower bound of $\mathcal{O}\left(10^{-7}\right)$. We see that lowering the upper bound on $\mathcal{B}(B \rightarrow K \mu \tau)$ at the LHCb and/or Belle II can have a major impact on the model building by further restraining the parameter space.

Scan of parameters with varying $\boldsymbol{m}_{\boldsymbol{U}_{1}}$. We now repeat the same analysis as before but by letting $m_{U_{1}}$ to be a free parameter too. $\chi_{\min }^{2}$ corresponds to the same couplings given in eq. (6.5), except that now $\chi^{2}$ is minimized along a line in parameter space defined by a constant $x_{L}^{i j} / m_{U_{1}}$, with $\left|x_{L}^{i j}\right|<\sqrt{4 \pi}$ (perturbativity limit). ${ }^{12}$ The main result of that analysis is shown in figure 10, where we show how the LFV branching fraction $\mathcal{B}(B \rightarrow K \mu \tau)$ (left panel) and $R_{D^{(*)}} / R_{D^{(*)}}^{\mathrm{SM}}$ (right panel) depend on $m_{U_{1}}$. We notice that, in order to be able to explain both flavor anomalies within the $U_{1}$ framework, $m_{U_{1}}$ cannot be higher than $\sim 12.5 \mathrm{TeV} .{ }^{13}$ This upper bound roughly corresponds to setting $x_{L}^{b \tau}=x_{L}^{s \tau}=\sqrt{4 \pi}$ and looking for the highest value of the mass for which the $b \rightarrow c \tau \nu$ anomaly can still be explained within $2 \sigma$. Moreover the lower bounds on both quantities remain rather stable when varying $m_{U_{1}}$.

Finally, the plots presented in figure 10 also showcase the complementarity between flavor physics (indirect) and LHC high- $p_{T}$ dilepton (direct) searches in constraining the $U_{1}$

\footnotetext{
${ }^{12}$ This scale invariance relationship holds in the case of $U_{1}$ because we only consider tree-level flavor constraints, and all the tree-level Wilson coefficients entering the analysis scale as (coupling/mass) ${ }^{2}$.

${ }^{13}$ Note that this value is similar to the upper bound derived from unitarity of $2 \rightarrow 2$ fermion scattering amplitudes, namely $\Lambda \approx 9 \mathrm{TeV}$ for the $b \rightarrow c$ transition [100] .
} 

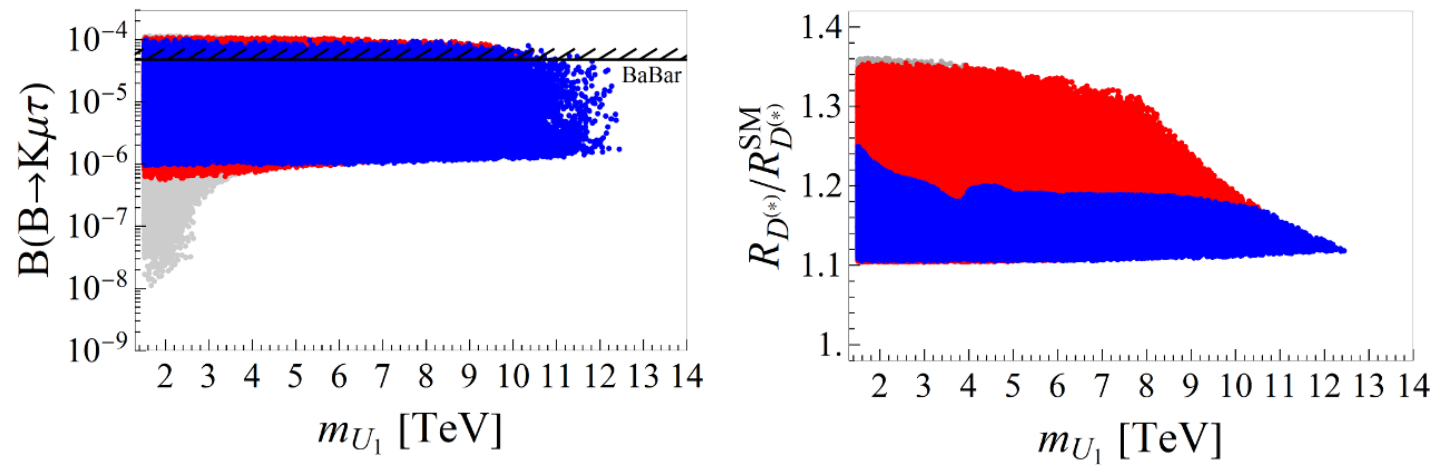

Figure 10. $m_{U_{1}}$ is plotted against $\mathcal{B}(B \rightarrow K \mu \tau)$ (left panel) and $R_{D^{(*)}} / R_{D^{(*)}}^{\mathrm{SM}}$ (right panel) for the $U_{1}$ model. Color code is the same as in figure 7 .

scenario as a possible explanation of the flavor anomalies. While accommodating $R_{K^{(*)}}<$ $R_{K^{(*)}}^{\mathrm{SM}}$ and $R_{D^{(*)}}>R_{D^{(*)}}^{\mathrm{SM}}$ already results in an absolute lower bound $\mathcal{B}(B \rightarrow K \mu \tau) \gtrsim 10^{-8}$, that bound gets shifted upwards by an order of magnitude to $\sim 5 \times 10^{-7}$ if one accounts for the direct LHC searches with $36 \mathrm{fb}^{-1}$ of data and for $m_{U_{1}}<4 \mathrm{TeV}$. Going to projected $300 \mathrm{fb}^{-1}$ leaves the lower bound on both $\mathcal{B}(B \rightarrow K \mu \tau)$ and $R_{D^{(*)}} / R_{D^{(*)}}^{\mathrm{SM}}$ quite stable. The upper bound on $\mathcal{B}(B \rightarrow K \mu \tau)$ is also stable but already superseded by the experimental limit on this decay mode established by BaBar. Measuring (bounding) $\mathcal{B}(B \rightarrow K \mu \tau)$ thus becomes very appealing as the improvement of the current upper bound can either help discarding the $U_{1}$ scenario altogether, or further corroborate its viability. Note that for definiteness we focus on $B \rightarrow K \mu \tau$, but the discussion would be completely equivalent if we discussed $B_{s} \rightarrow \mu \tau$ or $B \rightarrow K^{*} \mu \tau$, because their branching fractions are known to be related via $[24]$

$$
\frac{\mathcal{B}\left(B \rightarrow K^{*} \mu \tau\right)}{\mathcal{B}(B \rightarrow K \mu \tau)} \approx 1.8, \quad \frac{\mathcal{B}\left(B_{s} \rightarrow \mu \tau\right)}{\mathcal{B}(B \rightarrow K \mu \tau)} \approx 0.9
$$

This kind of LFV decay modes was also mentioned in refs. [101, 102] as a good probe of validity of a specific UV completion of the low-energy $U_{1}$ model.

As for the upper limit on $R_{D^{(*)}} / R_{D^{(*)}}^{\mathrm{SM}}$, we see that the direct searches can play a very important role in further reducing the space of parameters and with $300 \mathrm{fb}^{-1}$ of data at the LHC the possible range of values for this ratio reduces to $1.1 \leq R_{D^{(*)}} / R_{D^{(*)}}^{\mathrm{SM}} \leq 1.25$.

\section{Summary and conclusion}

In this work we revisited the single LQ solutions to the $B$-physics anomalies, $R_{D^{(*)}}^{\exp }>$ $R_{D^{(*)}}^{\mathrm{SM}}$ and/or $R_{K^{(*)}}^{\exp }<R_{K^{(*)}}^{\mathrm{SM}}$. We find that none of the scalar LQs alone, with the mass $m_{\mathrm{LQ}} \simeq 1 \mathrm{TeV}$, can provide a model of NP that accommodates simultaneously both kinds of anomalies. To arrive to that conclusion we combined a number of constraints on the model parameters arising from the low-energy flavor physics observables with those coming from the direct searches at the LHC. Concerning the latter ones the most significant constraints come from the large- $p_{T}$ spectrum of the differential cross section of $p p \rightarrow \ell \ell$. We use the 
most recent experimental results which we recast to obtain the bounds relevant to each of the models considered in this work. Since none of the scalar LQs can alone satisfy all the constraints (including the $B$-physics anomalies), see table 2, a promising route for model building involving leptoquarks seems to be combining two scalar LQs, in a way that has been done in refs. [88, 90, 91].

Besides the scalar LQs we also considered the vector ones. The main difficulty in this case is that one has to specify a UV completion of the model in order to compute the loop effects. By focusing only on the tree level observables, we confirm that the weak singlet vector $\mathrm{LQ}\left(U_{1}\right)$ of mass $m_{\mathrm{LQ}} \simeq 1 \div 2 \mathrm{TeV}$ can indeed accommodate both $R_{D^{(*)}}^{\exp }>R_{D^{(*)}}^{\mathrm{SM}}$ and $R_{K^{(*)}}^{\exp }<R_{K^{(*)}}^{\mathrm{SM}}$, in its minimal version, i.e. by allowing non-zero values only to the left-handed couplings [60]. We find that the new results from direct searches indeed push the lower bound of the vector LQ to larger values (above $1.5 \mathrm{TeV}$ ), and observe a pronounced complementarity of the low-energy (flavor physics) constraints with those obtained from direct searches. In particular we find the lower bound on the LFV mode $\mathcal{B}(B \rightarrow K \mu \tau) \gtrsim$ few $\times 10^{-7}$ for any mass of $m_{U_{1}}$ in which Yukawa couplings are kept within the perturbativity limits and in the minimal $U_{1}$ scenario in which only lefthanded couplings are allowed to take values different from zero. Notice that the upper bound is superseded by the current experimental bound $\mathcal{B}(B \rightarrow K \mu \tau)^{\exp }<4.8 \times 10^{-5}$, which can be improved both at LHCb and Belle II. Improving that bound by two orders of magnitude can therefore either exclude or, if observed, corroborate the validity of the minimal $U_{1}$ scenario.

\section{Acknowledgments}

This project has received support from the European Union's Horizon 2020 research and innovation programme under the Marie Sklodowska-Curie grant agreement $\mathrm{N}^{\circ} 674896$ and $\mathrm{N}^{\circ}$ 690575. Work at University of Nebraska-Lincoln is supported by the Department of Physics and Astronomy. A.A. would like to thank Frank Golf for insightful discussions on leptoquark searches at the LHC. D.A.F is supported by the Young Researchers Programme of the Slovenian Research Agency under the grant $N^{\circ} 37468$.

Open Access. This article is distributed under the terms of the Creative Commons Attribution License (CC-BY 4.0), which permits any use, distribution and reproduction in any medium, provided the original author(s) and source are credited.

\section{References}

[1] BaBAR collaboration, J.P. Lees et al., Evidence for an excess of $\bar{B} \rightarrow D^{(*)} \tau^{-} \bar{\nu}_{\tau}$ decays, Phys. Rev. Lett. 109 (2012) 101802 [arXiv:1205.5442] [INSPIRE].

[2] BABAR collaboration, J.P. Lees et al., Measurement of an Excess of $\bar{B} \rightarrow D^{(*)} \tau^{-} \bar{\nu}_{\tau}$ Decays and Implications for Charged Higgs Bosons, Phys. Rev. D 88 (2013) 072012 [arXiv: 1303.0571] [INSPIRE]. 
[3] BELLE collaboration, M. Huschle et al., Measurement of the branching ratio of $\bar{B} \rightarrow D^{(*)} \tau^{-} \bar{\nu}_{\tau}$ relative to $\bar{B} \rightarrow D^{(*)} \ell^{-} \bar{\nu}_{\ell}$ decays with hadronic tagging at Belle, Phys. Rev. D 92 (2015) 072014 [arXiv: 1507.03233] [INSPIRE].

[4] LHCb collaboration, Measurement of the ratio of branching fractions $\mathcal{B}\left(\bar{B}^{0} \rightarrow D^{*+} \tau^{-} \bar{\nu}_{\tau}\right) / \mathcal{B}\left(\bar{B}^{0} \rightarrow D^{*+} \mu^{-} \bar{\nu}_{\mu}\right)$, Phys. Rev. Lett. 115 (2015) 111803 [Erratum ibid. 115 (2015) 159901] [arXiv:1506.08614] [INSPIRE].

[5] Belle collaboration, S. Hirose et al., Measurement of the $\tau$ lepton polarization and $R\left(D^{*}\right)$ in the decay $\bar{B} \rightarrow D^{*} \tau^{-} \bar{\nu}_{\tau}$, Phys. Rev. Lett. 118 (2017) 211801 [arXiv:1612.00529] [INSPIRE].

[6] BeLLe collaboration, Y. Sato et al., Measurement of the branching ratio of $\bar{B}^{0} \rightarrow D^{*+} \tau^{-} \bar{\nu}_{\tau}$ relative to $\bar{B}^{0} \rightarrow D^{*+} \ell^{-} \bar{\nu}_{\ell}$ decays with a semileptonic tagging method, Phys. Rev. D 94 (2016) 072007 [arXiv: 1607.07923] [INSPIRE].

[7] BeLLe collaboration, A. Abdesselam et al., Measurement of the branching ratio of $\bar{B}^{0} \rightarrow D^{*+} \tau^{-} \bar{\nu}_{\tau}$ relative to $\bar{B}^{0} \rightarrow D^{*+} \ell^{-} \bar{\nu}_{\ell}$ decays with a semileptonic tagging method, arXiv: 1603.06711 [INSPIRE].

[8] HFLAV collaboration, Y. Amhis et al., Averages of b-hadron, c-hadron and $\tau$-lepton properties as of summer 2016, Eur. Phys. J. C 77 (2017) 895 [arXiv:1612.07233] [INSPIRE].

[9] MILC collaboration, J.A. Bailey et al., $B \rightarrow D \ell \nu$ form factors at nonzero recoil and $\left|V_{c b}\right|$ from $2+1$-flavor lattice QCD, Phys. Rev. D 92 (2015) 034506 [arXiv:1503.07237] [INSPIRE].

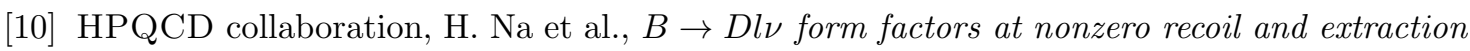
of $\left|V_{c b}\right|$, Phys. Rev. D 92 (2015) 054510 [Erratum ibid. D 93 (2016) 119906] [arXiv: 1505. 03925] [INSPIRE].

[11] S. Aoki et al., Review of lattice results concerning low-energy particle physics, Eur. Phys. J. C 77 (2017) 112 [arXiv: 1607.00299] [INSPIRE].

[12] D. Bigi, P. Gambino and S. Schacht, $R\left(D^{*}\right),\left|V_{c b}\right|$ and the Heavy Quark Symmetry relations between form factors, JHEP 11 (2017) 061 [arXiv:1707.09509] [INSPIRE].

[13] S. Jaiswal, S. Nandi and S.K. Patra, Extraction of $\left|V_{c b}\right|$ from $B \rightarrow D^{(*)} \ell \nu_{\ell}$ and the standard model predictions of $R\left(D^{(*)}\right)$, JHEP 12 (2017) 060 [arXiv:1707.09977] [INSPIRE].

[14] F.U. Bernlochner, Z. Ligeti, M. Papucci and D.J. Robinson, Combined analysis of semileptonic $B$ decays to $D$ and $D^{*}: R\left(D^{(*)}\right),\left|V_{c b}\right|$ and new physics, Phys. Rev. D 95 (2017) 115008 [Erratum ibid. D 97 (2018) 059902] [arXiv: 1703.05330] [INSPIRE].

[15] LHCb collaboration, Measurement of the ratio of branching fractions $\mathcal{B}\left(B_{c}^{+} \rightarrow J / \psi \tau^{+} \nu_{\tau}\right) / \mathcal{B}\left(B_{c}^{+} \rightarrow J / \psi \mu^{+} \nu_{\mu}\right)$, Phys. Rev. Lett. 120 (2018) 121801 [arXiv: 1711.05623] [INSPIRE].

[16] LHCb collaboration, Test of lepton universality using $B^{+} \rightarrow K^{+} \ell^{+} \ell^{-}$decays, Phys. Rev. Lett. 113 (2014) 151601 [arXiv: 1406.6482] [INSPIRE].

[17] LHCb collaboration, Test of lepton universality with $B^{0} \rightarrow K^{* 0} \ell^{+} \ell^{-}$decays, JHEP 08 (2017) 055 [arXiv: 1705. 05802] [inSPIRE].

[18] M. Bordone, G. Isidori and A. Pattori, On the standard model predictions for $R_{K}$ and $R_{K^{*}}$, Eur. Phys. J. C 76 (2016) 440 [arXiv:1605.07633] [InSPIRE]. 
[19] G. Hiller and F. Krüger, More model-independent analysis of $b \rightarrow s$ processes, Phys. Rev. D 69 (2004) 074020 [hep-ph/0310219] [INSPIRE].

[20] D. Bečirević, N. Košnik, O. Sumensari and R. Zukanovich Funchal, Palatable leptoquark scenarios for lepton flavor violation in exclusive $b \rightarrow s \ell_{1} \ell_{2}$ modes, JHEP 11 (2016) 035 [arXiv: 1608.07583] [INSPIRE].

[21] W. Altmannshofer, P. Bhupal Dev and A. Soni, $R_{D^{(*)}}$ anomaly: a possible hint for natural supersymmetry with R-parity violation, Phys. Rev. D 96 (2017) 095010 [arXiv: 1704.06659] [inSPIRE].

[22] S.L. Glashow, D. Guadagnoli and K. Lane, Lepton flavor violation in B decays?, Phys. Rev. Lett. 114 (2015) 091801 [arXiv: 1411.0565] [INSPIRE].

[23] C. Bobeth, M. Misiak and J. Urban, Photonic penguins at two loops and $m_{t}$ dependence of $B R\left[B \rightarrow X_{s} l^{+} l^{-}\right]$, Nucl. Phys. B 574 (2000) 291 [hep-ph/9910220] [INSPIRE].

[24] D. Bečirević, O. Sumensari and R. Zukanovich Funchal, Lepton flavor violation in exclusive $b \rightarrow s$ decays, Eur. Phys. J. C 76 (2016) 134 [arXiv: 1602.00881] [InSPIRE].

[25] B. Capdevila et al., Patterns of new physics in $b \rightarrow s \ell^{+} \ell^{-}$transitions in the light of recent data, JHEP 01 (2018) 093 [arXiv: 1704.05340] [INSPIRE].

[26] G. D'Amico et al., Flavour anomalies after the $R_{K^{*}}$ measurement, JHEP 09 (2017) 010 [arXiv: 1704.05438] [INSPIRE].

[27] W. Altmannshofer, P. Stangl and D.M. Straub, Interpreting hints for lepton flavor universality violation, Phys. Rev. D 96 (2017) 055008 [arXiv:1704.05435] [INSPIRE].

[28] T. Hurth, F. Mahmoudi, D. Martinez Santos and S. Neshatpour, Lepton nonuniversality in exclusive $b \rightarrow$ sll decays, Phys. Rev. D 96 (2017) 095034 [arXiv: 1705. 06274] [INSPIRE].

[29] LHCb collaboration, Measurement of the $B_{s}^{0} \rightarrow \mu^{+} \mu^{-}$branching fraction and effective lifetime and search for $B^{0} \rightarrow \mu^{+} \mu^{-}$decays, Phys. Rev. Lett. 118 (2017) 191801 [arXiv: 1703.05747] [INSPIRE].

[30] C. Bobeth et al., $B_{s, d} \rightarrow l^{+} l^{-}$in the standard model with reduced theoretical uncertainty, Phys. Rev. Lett. 112 (2014) 101801 [arXiv:1311.0903] [InSPIRE].

[31] I. Doršner et al., Physics of leptoquarks in precision experiments and at particle colliders, Phys. Rept. 641 (2016) 1 [arXiv:1603.04993] [InSPIRE].

[32] J. Aebischer, A. Crivellin, M. Fael and C. Greub, Matching of gauge invariant dimension-six operators for $b \rightarrow s$ and $b \rightarrow c$ transitions, JHEP 05 (2016) 037 [arXiv: 1512.02830] [INSPIRE].

[33] M. Jung and D.M. Straub, Constraining new physics in $b \rightarrow c \ell \nu$ transitions, arXiv: 1801.01112 [INSPIRE].

[34] M. Freytsis, Z. Ligeti and J.T. Ruderman, Flavor models for $\bar{B} \rightarrow D^{(*)} \tau \bar{\nu}$, Phys. Rev. D 92 (2015) 054018 [arXiv: 1506.08896] [inSPIRE].

[35] D. Bečirević, B. Panes, O. Sumensari and R. Zukanovich Funchal, Seeking leptoquarks in IceCube, JHEP 06 (2018) 032 [arXiv:1803.10112] [INSPIRE].

[36] D. Bečirević, N. Košnik and A. Tayduganov, $\bar{B} \rightarrow D \tau \bar{\nu}_{\tau}$ vs. $\bar{B} \rightarrow D \mu \bar{\nu}_{\mu}$, Phys. Lett. B 716 (2012) 208 [arXiv: 1206.4977] [InSPIRE].

[37] A. Celis, M. Jung, X.-Q. Li and A. Pich, Scalar contributions to $b \rightarrow c(u) \tau \nu$ transitions, Phys. Lett. B 771 (2017) 168 [arXiv:1612.07757] [INSPIRE]. 
[38] A. Celis, M. Jung, X.-Q. Li and A. Pich, Sensitivity to charged scalars in $B \rightarrow D^{(*)} \tau \nu_{\tau}$ and $B \rightarrow \tau \nu_{\tau}$ decays, JHEP 01 (2013) 054 [arXiv: 1210.8443] [INSPIRE].

[39] P. Biancofiore, P. Colangelo and F. De Fazio, On the anomalous enhancement observed in $B \rightarrow D^{(*)} \tau \bar{\nu}_{\tau}$ decays, Phys. Rev. D 87 (2013) 074010 [arXiv:1302.1042] [INSPIRE].

[40] P. Colangelo and F. De Fazio, Scrutinizing $\bar{B} \rightarrow D^{*}(D \pi) \ell^{-} \bar{\nu}_{\ell}$ and $\bar{B} \rightarrow D^{*}(D \gamma) \ell^{-} \bar{\nu}_{\ell}$ in search of new physics footprints, JHEP 06 (2018) 082 [arXiv:1801.10468] [INSPIRE].

[41] F. Feruglio, P. Paradisi and O. Sumensari, Implications of scalar and tensor explanations of $R_{D^{(*)}}$, arXiv: 1806.10155 [INSPIRE].

[42] M.A. Ivanov, J.G. Körner and C.-T. Tran, Probing new physics in $\bar{B}^{0} \rightarrow D^{(*)} \tau^{-} \bar{\nu}_{\tau}$ using the longitudinal, transverse and normal polarization components of the $\tau$ lepton, Phys. Rev. D 95 (2017) 036021 [arXiv: 1701.02937] [INSPIRE].

[43] A. Azatov et al., Anatomy of $b \rightarrow c \tau \nu$ anomalies, arXiv:1805.03209 [INSPIRE].

[44] Z.-R. Huang et al., Footprints of new physics in $b \rightarrow c \tau \nu$ transitions, arXiv:1808.03565 [INSPIRE].

[45] M. González-Alonso, J. Martin Camalich and K. Mimouni, Renormalization-group evolution of new physics contributions to (semi)leptonic meson decays, Phys. Lett. B 772 (2017) 777 [arXiv: 1706.00410] [INSPIRE].

[46] X.-Q. Li, Y.-D. Yang and X. Zhang, Revisiting the one leptoquark solution to the $R\left(D^{(*)}\right)$ anomalies and its phenomenological implications, JHEP 08 (2016) 054 [arXiv:1605.09308] [INSPIRE].

[47] R. Alonso, B. Grinstein and J. Martin Camalich, Lifetime of $B_{c}^{-}$constrains explanations for anomalies in $B \rightarrow D^{(*)} \tau \nu$, Phys. Rev. Lett. 118 (2017) 081802 [arXiv:1611.06676] [INSPIRE].

[48] I. Doršner, S. Fajfer, D.A. Faroughy and N. Košnik, The role of the $S_{3}$ GUT leptoquark in flavor universality and collider searches, arXiv:1706.07779 [INSPIRE].

[49] G. Hiller and M. Schmaltz, $R_{K}$ and future $b \rightarrow$ sll physics beyond the standard model opportunities, Phys. Rev. D 90 (2014) 054014 [arXiv:1408.1627] [INSPIRE].

[50] G. Hiller and I. Nisandzic, $R_{K}$ and $R_{K^{*}}$ beyond the standard model, Phys. Rev. D 96 (2017) 035003 [arXiv: 1704.05444] [inSPIRE].

[51] C. Hati, G. Kumar, J. Orloff and A.M. Teixeira, Reconciling B-decay anomalies with neutrino masses, dark matter and constraints from flavour violation, arXiv:1806.10146 [INSPIRE].

[52] D. Bečirević and O. Sumensari, A leptoquark model to accommodate $R_{K}^{\exp }<R_{K}^{\mathrm{SM}}$ and $R_{K^{*}}^{\exp }<R_{K^{*}}^{\mathrm{SM}}$, JHEP 08 (2017) 104 [arXiv: 1704.05835] [INSPIRE].

[53] N. Assad, B. Fornal and B. Grinstein, Baryon number and lepton universality violation in leptoquark and diquark models, Phys. Lett. B 777 (2018) 324 [arXiv:1708.06350] [INSPIRE].

[54] Y. Sakaki, M. Tanaka, A. Tayduganov and R. Watanabe, Testing leptoquark models in $\bar{B} \rightarrow D^{(*)} \tau \bar{\nu}$, Phys. Rev. D 88 (2013) 094012 [arXiv:1309.0301] [INSPIRE].

[55] G. Hiller, D. Loose and K. Schönwald, Leptoquark flavor patterns $\mathcal{E} 3$ b decay Anomalies, JHEP 12 (2016) 027 [arXiv:1609.08895] [INSPIRE]. 
[56] D. Bečirević, S. Fajfer and N. Košnik, Lepton flavor nonuniversality in $b \rightarrow s \ell^{+} \ell^{-}$ processes, Phys. Rev. D 92 (2015) 014016 [arXiv: 1503.09024] [INSPIRE].

[57] P. Cox, A. Kusenko, O. Sumensari and T.T. Yanagida, SU(5) unification with TeV-scale leptoquarks, JHEP 03 (2017) 035 [arXiv: 1612.03923] [INSPIRE].

[58] D. Bečirević, S. Fajfer, N. Košnik and O. Sumensari, Leptoquark model to explain the $B$-physics anomalies, $R_{K}$ and $R_{D}$, Phys. Rev. D 94 (2016) 115021 [arXiv:1608.08501] [INSPIRE].

[59] M. Bauer and M. Neubert, Minimal leptoquark explanation for the $R_{D^{(*)}}, R_{K}$ and $(g-2)_{g}$ anomalies, Phys. Rev. Lett. 116 (2016) 141802 [arXiv:1511.01900] [INSPIRE].

[60] D. Buttazzo, A. Greljo, G. Isidori and D. Marzocca, B-physics anomalies: a guide to combined explanations, JHEP 11 (2017) 044 [arXiv: 1706.07808] [INSPIRE].

[61] L. Di Luzio, A. Greljo and M. Nardecchia, Gauge leptoquark as the origin of B-physics anomalies, Phys. Rev. D 96 (2017) 115011 [arXiv:1708.08450] [INSPIRE].

[62] M. Bordone, C. Cornella, J. Fuentes-Martin and G. Isidori, A three-site gauge model for flavor hierarchies and flavor anomalies, Phys. Lett. B 779 (2018) 317 [arXiv:1712.01368] [INSPIRE].

[63] L. Calibbi, A. Crivellin and T. Li, A model of vector leptoquarks in view of the B-physics anomalies, arXiv: 1709.00692 [INSPIRE].

[64] M. Blanke and A. Crivellin, B meson anomalies in a Pati-Salam model within the Randall-Sundrum background, Phys. Rev. Lett. 121 (2018) 011801 [arXiv:1801.07256] [INSPIRE].

[65] R. Barbieri and A. Tesi, B-decay anomalies in Pati-Salam SU(4), Eur. Phys. J. C 78 (2018) 193 [arXiv: 1712.06844] [INSPIRE].

[66] A. Greljo and B.A. Stefanek, Third family quark-lepton unification at the TeV scale, Phys. Lett. B 782 (2018) 131 [arXiv: 1802.04274] [INSPIRE].

[67] S. Fajfer and N. Košnik, Vector leptoquark resolution of $R_{K}$ and $R_{D^{(*)}}$ puzzles, Phys. Lett. B 755 (2016) 270 [arXiv:1511.06024] [INSPIRE].

[68] I. Dorsner, S. Fajfer and A. Greljo, Cornering scalar leptoquarks at LHC, JHEP 10 (2014) 154 [arXiv: 1406.4831] [INSPIRE].

[69] CMS collaboration, Search for third-generation scalar leptoquarks and heavy right-handed neutrinos in final states with two tau leptons and two jets in proton-proton collisions at $\sqrt{s}=13 \mathrm{TeV}$, JHEP 07 (2017) 121 [arXiv:1703.03995] [INSPIRE].

[70] CMS collaboration, Search for third-generation scalar leptoquarks decaying to a top quark and a $\tau$ lepton at $\sqrt{s}=13 \mathrm{TeV}$, Eur. Phys. J. C 78 (2018) 707 [arXiv:1803.02864] [INSPIRE].

[71] CMS Collaboration, Search for pair production of second generation leptoquarks at $\sqrt{s}=13$ $\mathrm{TeV}$, CMS-PAS-EXO-17-003 (2017).

[72] B. Diaz, M. Schmaltz and Y.-M. Zhong, The leptoquark Hunter's guide: pair production, JHEP 10 (2017) 097 [arXiv:1706. 05033] [INSPIRE].

[73] J.E. Camargo-Molina, A. Celis and D.A. Faroughy, Anomalies in bottom from new physics in top, Phys. Lett. B 784 (2018) 284 [arXiv: 1805. 04917] [INSPIRE]. 
[74] CMS collaboration, Search for leptoquarks coupling to third generation quarks, CMS-PAS-B2G-16-027 (2016).

[75] CMS collaboration, Constraints on models of scalar and vector leptoquarks decaying to a quark and a neutrino at $\sqrt{s}=13 \mathrm{TeV}$, CMS-PAS-SUS-18-001 (2018).

[76] J. Blumlein, E. Boos and A. Kryukov, Leptoquark pair production in hadronic interactions, Z. Phys. C 76 (1997) 137 [hep-ph/9610408] [INSPIRE].

[77] D.A. Faroughy, A. Greljo and J.F. Kamenik, Confronting lepton flavor universality violation in $B$ decays with high- $p_{T}$ tau lepton searches at LHC, Phys. Lett. B 764 (2017) 126 [arXiv:1609.07138] [INSPIRE].

[78] A. Greljo and D. Marzocca, High-p $p_{T}$ dilepton tails and flavor physics, Eur. Phys. J. C 77 (2017) 548 [arXiv:1704.09015] [INSPIRE].

[79] ATLAS collaboration, Search for new high-mass phenomena in the dilepton final state using $36 \mathrm{fb}^{-1}$ of proton-proton collision data at $\sqrt{\mathrm{s}}=13$ TeV with the ATLAS detector, JHEP 10 (2017) 182 [arXiv:1707.02424] [INSPIRE].

[80] ATLAS collaboration, Search for additional heavy neutral Higgs and gauge bosons in the ditau final state produced in $36 \mathrm{fb}^{-1}$ of pp collisions at $\sqrt{\mathrm{s}}=13 \mathrm{TeV}$ with the ATLAS detector, JHEP 01 (2018) 055 [arXiv: 1709.07242] [INSPIRE].

[81] G. Cowan, K. Cranmer, E. Gross and O. Vitells, Asymptotic formulae for likelihood-based tests of new physics, Eur. Phys. J. C 71 (2011) 1554 [Erratum ibid. C 73 (2013) 2501] [arXiv:1007.1727] [INSPIRE].

[82] A. Alloul et al., FeynRules 2.0 - A complete toolbox for tree-level phenomenology, Comput. Phys. Commun. 185 (2014) 2250 [arXiv:1310.1921] [INSPIRE].

[83] J. Alwall et al., The automated computation of tree-level and next-to-leading order differential cross sections and their matching to parton shower simulations, JHEP 07 (2014) 079 [arXiv: 1405.0301] [INSPIRE].

[84] T. Sjöstrand et al., An introduction to PYTHIA 8.2, Comput. Phys. Commun. 191 (2015) 159 [arXiv: 1410.3012] [INSPIRE].

[85] DELPHES 3 collaboration, J. de Favereau et al., DELPHES 3, a modular framework for fast simulation of a generic collider experiment, JHEP 02 (2014) 057 [arXiv:1307.6346] [INSPIRE].

[86] ATLAS collaboration, Search for new phenomena in different-flavour high-mass dilepton final states in $p p$ collisions at $\sqrt{s}=13$ TeV with the ATLAS detector, Eur. Phys. J. C 76 (2016) 541 [arXiv:1607.08079] [INSPIRE].

[87] ATLAS collaboration, Search for lepton-flavor violation in different-flavor, high-mass final states in pp collisions at $\sqrt{s}=13$ TeV with the ATLAS detector, submitted to Phys. Rev., arXiv: 1807.06573 [INSPIRE].

[88] D. Bečirević et al., Scalar leptoquarks from grand unified theories to accommodate the B-physics anomalies, Phys. Rev. D 98 (2018) 055003 [arXiv: 1806.05689] [INSPIRE].

[89] Y. Cai, J. Gargalionis, M.A. Schmidt and R.R. Volkas, Reconsidering the One Leptoquark solution: flavor anomalies and neutrino mass, JHEP 10 (2017) 047 [arXiv: 1704.05849] [INSPIRE]. 
[90] D. Marzocca, Addressing the B-physics anomalies in a fundamental Composite Higgs Model, JHEP 07 (2018) 121 [arXiv:1803.10972] [INSPIRE].

[91] A. Crivellin, D. Müller and T. Ota, Simultaneous explanation of $R\left(D^{(*)}\right)$ and $b \rightarrow s \mu^{+} \mu^{-}$: the last scalar leptoquarks standing, JHEP 09 (2017) 040 [arXiv:1703.09226] [INSPIRE].

[92] Particle Data Group collaboration, C. Patrignani et al., Review of particle physics, Chin. Phys. C 40 (2016) 100001.

[93] BelLe collaboration, Y. Miyazaki et al., Search for lepton-flavor-violating $\tau$ decays into a lepton and a vector meson, Phys. Lett. B 699 (2011) 251 [arXiv:1101.0755] [INSPIRE].

[94] F. Feruglio, P. Paradisi and A. Pattori, Revisiting lepton flavor universality in B decays, Phys. Rev. Lett. 118 (2017) 011801 [arXiv:1606.00524] [INSPIRE].

[95] F. Feruglio, P. Paradisi and A. Pattori, On the importance of electroweak corrections for $B$ anomalies, JHEP 09 (2017) 061 [arXiv: 1705. 00929] [INSPIRE].

[96] C. Cornella, F. Feruglio and P. Paradisi, Low-energy effects of lepton flavour universality violation, arXiv: 1803.00945 [INSPIRE].

[97] V. Cirigliano and I. Rosell, Two-loop effective theory analysis of $\pi(K) \rightarrow e \bar{\nu} e[\gamma]$ branching ratios, Phys. Rev. Lett. 99 (2007) 231801 [arXiv:0707.3439] [INSPIRE].

[98] Belle collaboration, R. Glattauer et al., Measurement of the decay $B \rightarrow D \ell \nu_{\ell}$ in fully reconstructed events and determination of the Cabibbo-Kobayashi-Maskawa matrix element $\left|V_{c b}\right|$, Phys. Rev. D 93 (2016) 032006 [arXiv: 1510.03657] [INSPIRE].

[99] BABAR collaboration, J.P. Lees et al., A search for the decay modes $B^{ \pm} \rightarrow h^{ \pm} \tau^{ \pm} l$, Phys. Rev. D 86 (2012) 012004 [arXiv:1204.2852] [INSPIRE].

[100] L. Di Luzio and M. Nardecchia, What is the scale of new physics behind the B-flavour anomalies?, Eur. Phys. J. C 77 (2017) 536 [arXiv:1706.01868] [INSPIRE].

[101] M. Bordone, C. Cornella, J. Fuentes-Martín and G. Isidori, Low-energy signatures of the $P S^{3}$ model: from B-physics anomalies to LFV, arXiv:1805.09328 [INSPIRE].

[102] L. Di Luzio, J. Fuentes-Martin, A. Greljo, M. Nardecchia and S. Renner, Maximal Flavour Violation: a Cabibbo mechanism for leptoquarks, arXiv:1808.00942 [INSPIRE]. 